\title{
Analysis of TPC Single Sextant U-238/U-235 Engineering In-Beam Data
}

September 2012

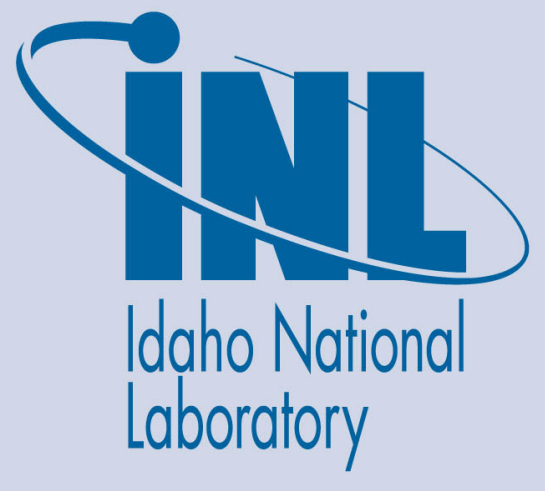

The INL is a U.S. Department of Energy National Laboratory operated by Battelle Energy Alliance 
INL/EXT-12-27395

\title{
Analysis of TPC Single Sextant U-238/U-235 Engineering In-Beam Data
}

September 2012

\author{
Idaho National Laboratory \\ Idaho Falls, Idaho 83415
}

http://www.inl.gov

Prepared for the

U.S. Department of Energy

Office of Nuclear Energy

Under DOE Idaho Operations Office

Contract DE-AC07-05ID14517 


\section{Analysis of TPC Single Sextant U-238/U-235 Engineering In-Beam Data}

Deliverable for M2FT-12IN0210043

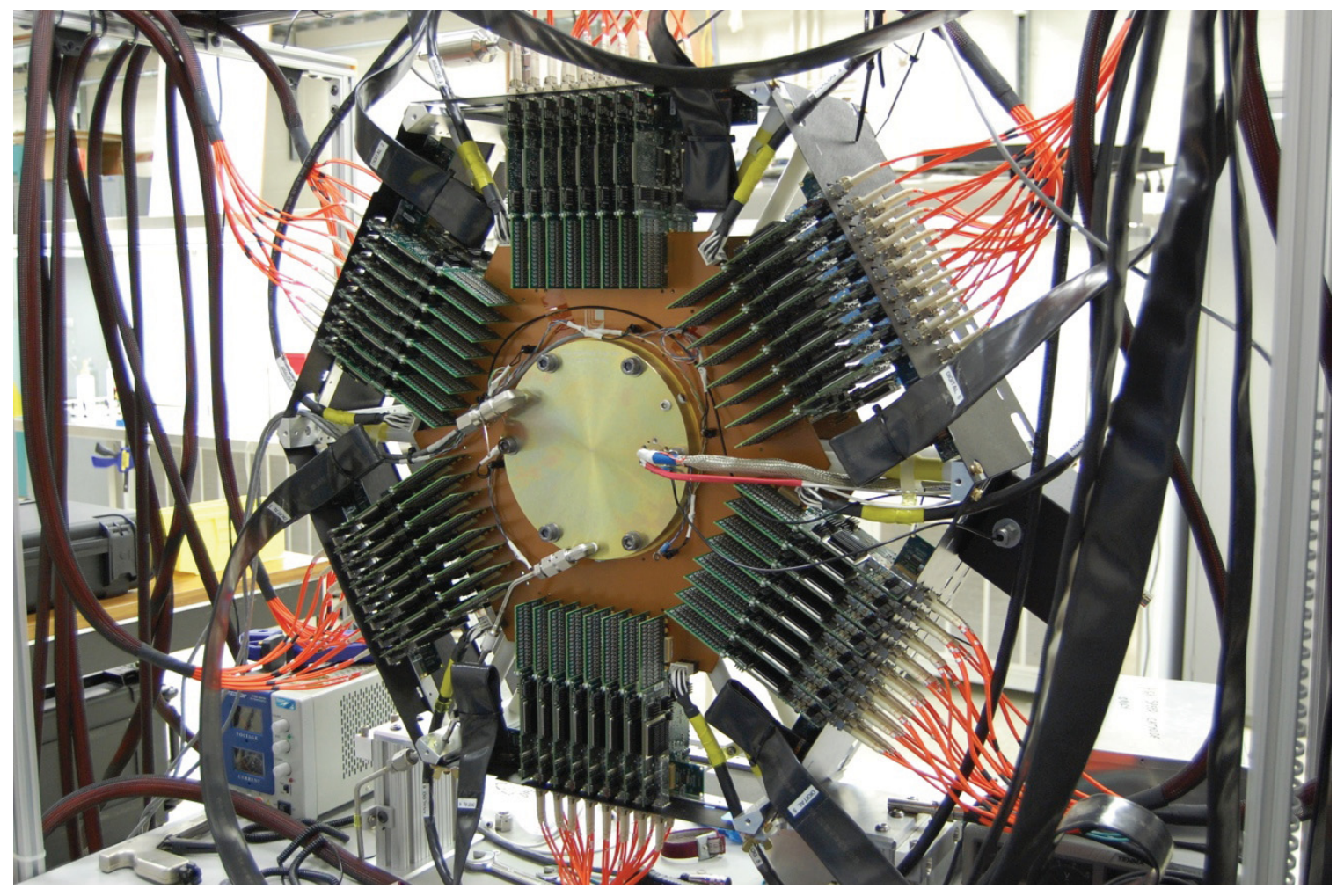

The Time Projection Chamber is a collaborative effort to implement an innovative approach and deliver unprecedented fission measurements to DOE programs. This $4 \pi$-detector system will provide unrivaled 3-D data about the fission process. Shown here is a half populated TPC (six of twelve sextants) on the test stand at the LLNL TPC laboratory. This TPC has been shipped and installed at LANSCE and is collecting further engineering data for the full system scale up next year. 


\section{Blank Page}




\section{TPC Project Timeline}
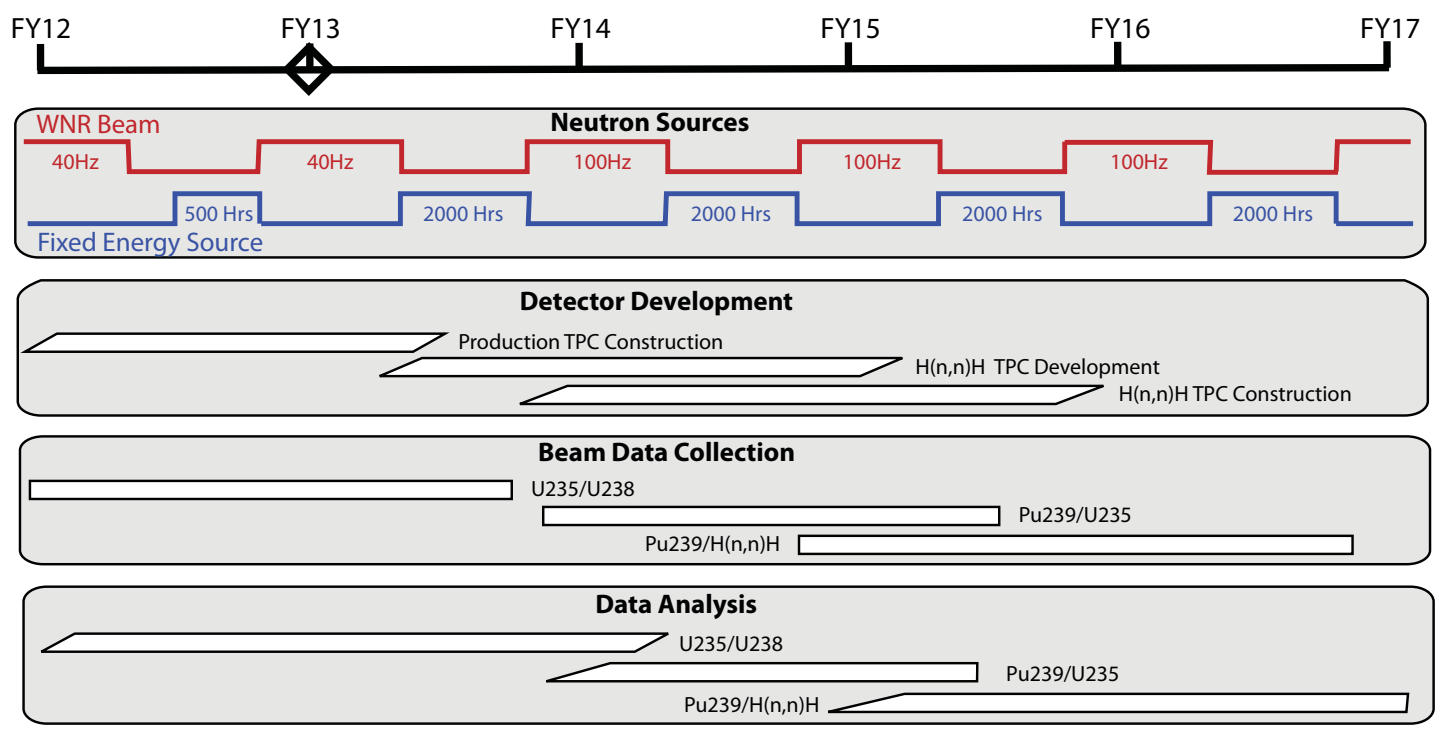

\section{Major Milestones/Deliverables Timeline}

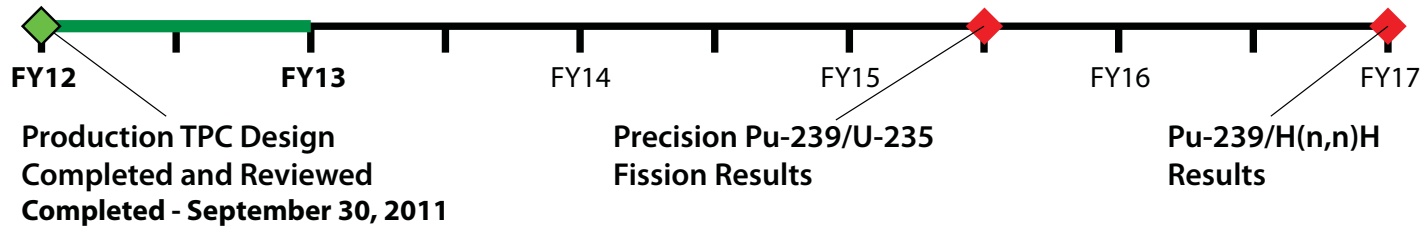

\section{FY12 Supporting Milestones/Deliverables}

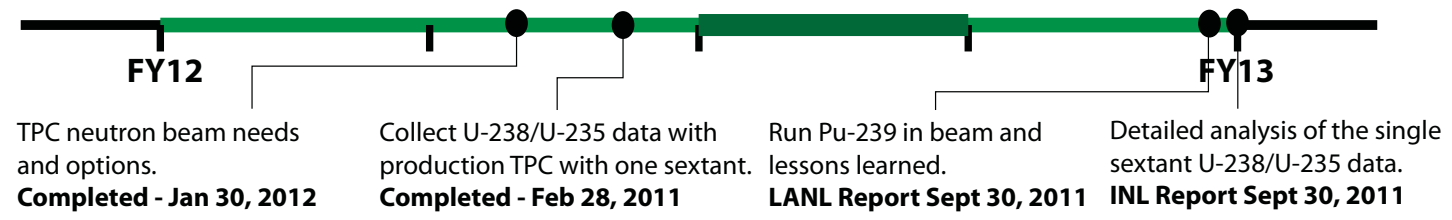

\section{Fourth Quarter Highlights FY12}

- The scale-up to half an instrumented TPC has been successful, including the integration of the highspeed cathode readout system. The system is being tested in beam at LANSCE.

- The University funding and participation was restored last quarter after lenghty funding delays.

-A second Ph.D. was granted in the TPC project, to Lucas Snyder of the Colorado School of Mines, for his alpha to fission rate ratio measurement of Cf-252 using data from the single sectant configuration.

\section{Potential Timeline Issues and Concerns}

- Delayed funding for the University contract resulted in a lapse of support for several months this year. As a result, software development task efforts will be increased in the coming months. University funding remains a challenge in reduced budget scenario with future funding unclear.

- $100 \mathrm{~Hz}$ operations at the LANSCE accelerator have not been recovered yet and the schedule is unclear. 


\section{Blank Page}




\section{Table of Contents}

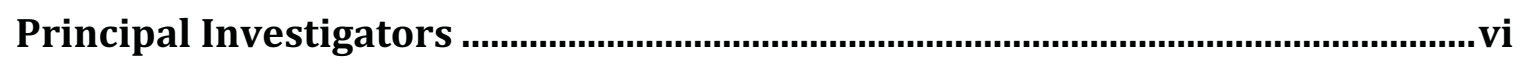

Acronyms and Symbols................................................................................ vii

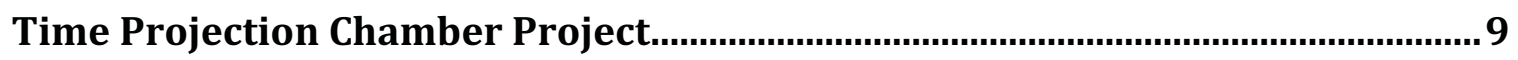

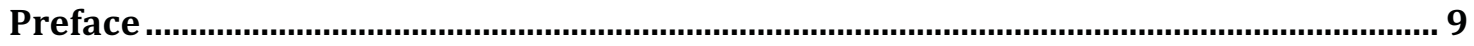

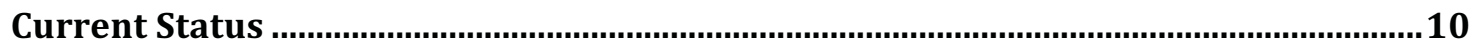

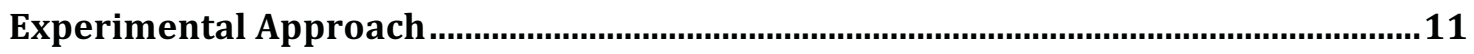

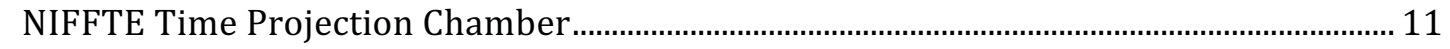

Los Alamos Neutron Science Center .................................................................................................. 14

Experiment................................................................................................16

Run1 : U238+U235 - November 29 - December 17, 2011 .......................................................16

Run2 : U238+U235 - January 12 through 27, 2012 ……………………………………….... 18

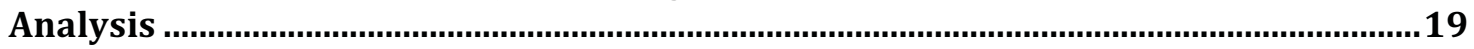

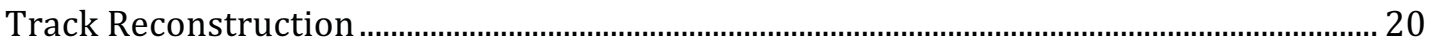

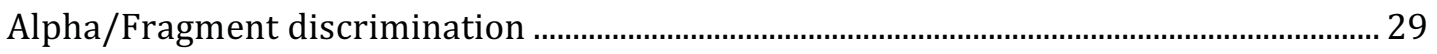

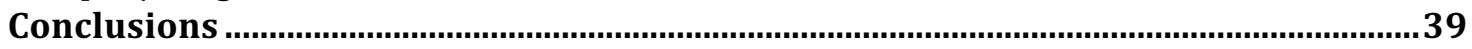




\section{Principal Investigators}

Universities

NE funded

E. Burgett

J. Klay

R. Towell

Idaho State University

D. Isenhower

U. Greife

W. Loveland

S. Grimes

T. Massey

California Polytechnic State University, San Luis Obispo

Abilene Christian University

Abilene Christian University

Colorado School of Mines

Oregon State University

Ohio University

Ohio University

\section{Laboratories}

NE, NNSA funded

T. Hill

Idaho National Laboratory

M. Heffner

F. Tovesson

Lawrence Livermore National Laboratory

Los Alamos National Laboratory

D. Asner

Pacific Northwest National Laboratory 


\section{Acronyms and Symbols}

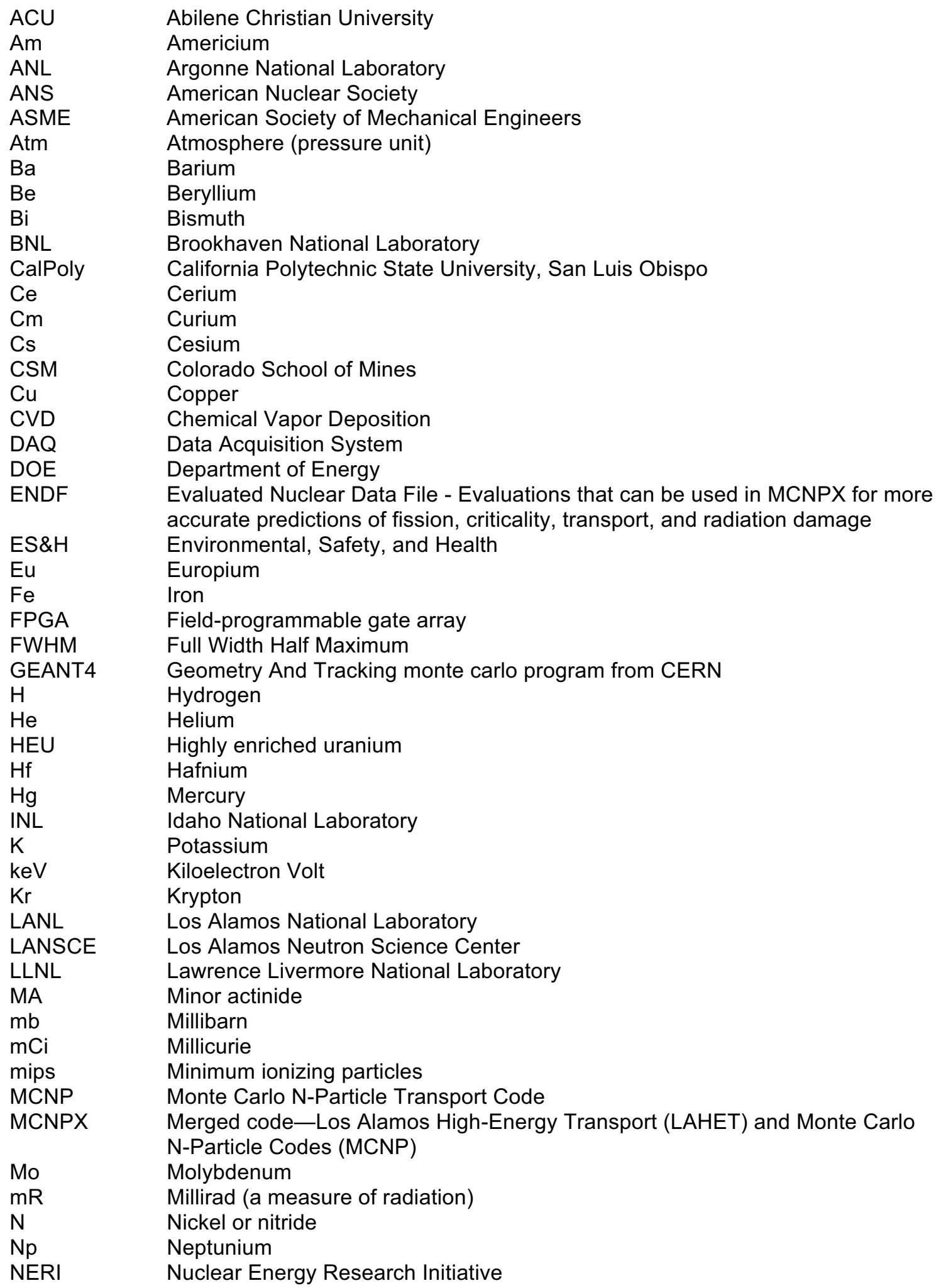




$\begin{array}{ll}\text { NIFFTE } & \text { Neutron Induced Fission Fragment Tracking Experiment (TPC Collaboration } \\ \text { name) } \\ \text { O } & \text { Oxygen or Oxide } \\ \text { ORNL } & \text { Oak Ridge National Laboratory } \\ \text { OSU } & \text { Oregon State University } \\ \text { OU } & \text { The Ohio University } \\ \text { PACS } & \text { Personnel Access Control System } \\ \text { Pb } & \text { Lead } \\ \text { Pd } & \text { Paladium } \\ \text { PNNL } & \text { Pacific Northwest National Laboratory } \\ \text { Pu } & \text { Plutonium } \\ \text { QA } & \text { Quality Assurance } \\ \text { R } & \text { Rad (a measure of radiation) } \\ \text { rms } & \text { root mean square } \\ \text { ROOT } & \text { an object oriented data analysis framework from CERN } \\ \text { Ru } & \text { Ruthenium } \\ \text { SEM } & \text { Scanning Electron Microscopy } \\ \text { STP } & \text { Standard Temperature and Pressure } \\ \text { Ta } & \text { Tantalum } \\ \text { TC } & \text { Technitium } \\ \text { TPC } & \text { Time Projection Chamber } \\ \text { U } & \text { Uranium } \\ \text { V } & \text { Vanadium } \\ \text { W } & \text { Tungsten } \\ \text { WBS } & \text { Work Breakdown Structure } \\ \text { WNR } & \text { Weapons Neutron Research (facility at LANSCE) } \\ \text { Xe } & \text { Xenon } \\ \text { Y } & \text { Yttrium } \\ \text { Zr } & \text { Zirconium }\end{array}$




\section{Time Projection Chamber Project}

\section{Preface}

Reactors, weapons and nucelo-synthesis calculations are all dependent on nuclear physics for cross sections and particle kinematics. These applications are very sensitive to the nuclear physics in the fast neutron energy region and therefore have large overlaps in nuclear data needs. High performance computer codes interface the nuclear data through nuclear data libraries, which are a culmination of experimental results and nuclear theory and modeling. Uncertainties in the data contained in those libraries propagate into uncertainties in calculated performance parameters. The impact of nuclear data uncertainties has been studied in detail for reactor and weapon systems and sensitivity codes have subsequently been developed that provide nuclear data accuracy requirements based on adopted target accuracies on crucial design parameters. The sensitivity calculations have been performed for a number of candidate systems. These sensitivity studies provide specific requirements for uncertainties on many fission cross sections, many of which are beyond the reach of current experimental tools. The sensitivity codes are proving to be very useful for identifying the highest impact measurements for DOE programs and the TPC measurement program will help provide those data. The result of these new, high-accuracy precision measurements will be a refined understanding of performance results, thus reducing the liability nuclear data has on the overall uncertainties in calculated integral quantities. The new class of high-accuracy, highprecision fission measurements will not be easy. The proposed method is to employ a Time Projection Chamber and perform fission measurements relative to $H(n, n) H$ elastic scattering. The TPC technology has been in use in high-energy physics for over two decades - it is well developed and well understood. However, it will have to be optimized for this task that includes miniaturization, design for hydrogen gas, and large dynamic range electronics. The TPC is the perfect tool for minimizing most of the systematic errors associated with typical fission measurements. The idea is to engineer a TPC specifically for delivering fission cross section measurements with uncertainties below $1.0 \%$.

The long term goal is to fill the TPC with hydrogen gas and measure fission cross sections relative to $\mathrm{H}(n, n) \mathrm{H}$ elastic scattering, thus removing the uncertainties associated with using the U-235 fission cross section for normalization. In fact, we will provide the world's best differential measurement of the U-235 fission cross section and this will impact nearly all fission library data, since it has been used as a standard in much of the available fission experimental data.

The immediate objective of this effort is to implement a fission cross section measurement program with the goal of providing the most needed measurements with unprecedented precision and accuracy using a time projection chamber. Previous measurements of fission cross sections have been hampered primarily by three systematic uncertainties that cannot be overcome using standard techniques. The first systematic error to address is the misidentification of fission products and alpha decays, particularly in the highly radioactive environment of Pu-239. By using full track reconstruction, the TPC will provide unambiguous identification of all 
charged particles in the chamber. The second systematic to overcome is the target and beam non-uniformities that are typically convolved in previous experiments. The TPC provides precise tracking information on the origin of each track as it emerges from the target material, thus enabling an autoradiograph of the material, along with the neutron beam profile as a function of position. The third systematic uncertainty to be addressed is that of the standard reference material. Previous experiments have relied on the $U-235$ cross section for normalization, which limits the overall accuracy of any relative measurement to the U-235 uncertainties, which are of order 1 to $2 \%$. The TPC has been designed to use the hydrogen gas in the active volume in order to normalize the TPC data to the much better known elastic scattering cross section of neutrons on protons. The reduction in uncertainties by addressing all three of the primary systematic uncertainties will provide differential fission cross section measurement to the sub-percent level, as required by sensitivity studies of fast neutron systems.

The first three years of this program will provide all the groundwork and infrastructure for a successful measurement campaign. Shortly following, we will provide precision fission ratio measurements for $\mathrm{Pu}-239 / \mathrm{U}-235$ and $\mathrm{U}-238 / \mathrm{U}-235$ along with a full design proposal to measure ${ }^{235} \mathrm{U} / \mathrm{n}(\mathrm{n}, \mathrm{p}) \mathrm{p}$. The ${ }^{235} \mathrm{U} / \mathrm{H}(\mathrm{n}, \mathrm{n}) \mathrm{H}$ measurement will provide the best single measurement of the U-235 fission cross section and will allow us to convert the initial, and any subsequent, ratio experiments to worlds best absolute measurements. After completion of the U-238 and Pu-239 ratio measurements, the experimenters will move on to measurement of the minor actinide cross sections, fission fragment distribution and neutron yield measurements. This information will play a crucial role in the long term DOE nuclear R\&D campaigns.

\section{Current Status}

The current TPC is collecting neutron beam data at the LANSCE WNR facility in Los Alamos with approximately half of the detector instrumented. The system is operating at expected performance levels based on the development approach adopted for this effort. The TPC is being tested during the development phase to insure that the required performance goals can be met in the LANSCE beam environment, something that is impossible to simulate on a test bench. This approach has been very fruitful in providing timely and important performance feedback for hardware and software development. This report is focused on the performance of the TPC during data collection of U-235 and U-238 data from a single target but includes data from other beam experiments in the R\&D program along with bench test results. The two uranium isotopes in the primary experiment were deposited on a single target in order to collect fission data from both isotopes simultaneously during beam runs early in FY12. The decision to use a segmented target provides not only fission data for both isotopes simultaneously but allows for testing of the basic tracking capabilities in separating their positions on the target. The ultimate pointing resolution of the TPC is dependent on a fast timing signal that denotes the beginning of the event and that system has been installed and is collecting data at the writing of this report. The TPC configuration was limited in instrumentation to one-sixth of the collection volume in previous tests to assure that the power and signal routing hardware performed as expected before scaling up to a fully instrumented device (a half instrumented TPC is collecting data at LANSCE during the writing of this report). As such, the capabilities of the partially instrumented 
TPC is limited and complicated. The limited viewing volume contains not only fission products and alpha decays that were fully contained in that volume but is complicated by partial tracks that either left the field of view or entered it from outside. The overall TPC performance is also hampered in analysis by a lack of any, let alone precise, channel by channel calibration data or the deconvolution of detector behaviors, such as cross talk and charge diffusion. However, it is clear from the initial detailed analyses, that the particle identification information and vertexing capabilities will meet the needs for reducing or removing these particular systematic uncertainties in the actual production measurements to be carried out in future years.

\section{Experimental Approach}

\section{NIFFTE Time Projection Chamber}

Time Projection Chambers (TPCs) have been used in high-energy physics for decades - the NIFFTE TPC, however, marks the first application of TPC technology to fission research. The challenge of bringing this technology to fission measurements lies in miniaturizing the system appropriately, with special attention to signal fidelity and high bandwidth requirements. The design of such a system has only recently been possible with advances in micro-electronics and modern computing power. The TPC collects nearly half a terabyte of data per second that is processed to a manageable flow in a parallel computing paradigm and within inches of the experiment. The detection principles of TPCs are illustrated in Figure 1.

Charged particles passing through a fill gas produce ionization electrons, which drift through a uniform electric field towards a highly segmented readout plane. The segmented readout allows one to reconstruct a two-dimensional projection of the particle track. The signals from each pad are digitized as the incoming charge accumulates as a function of time using synchronized clocks. Combining the physical two-dimensional projection with the drift time of the electrons provides a full, threedimensional picture of the particle's path through the detector.

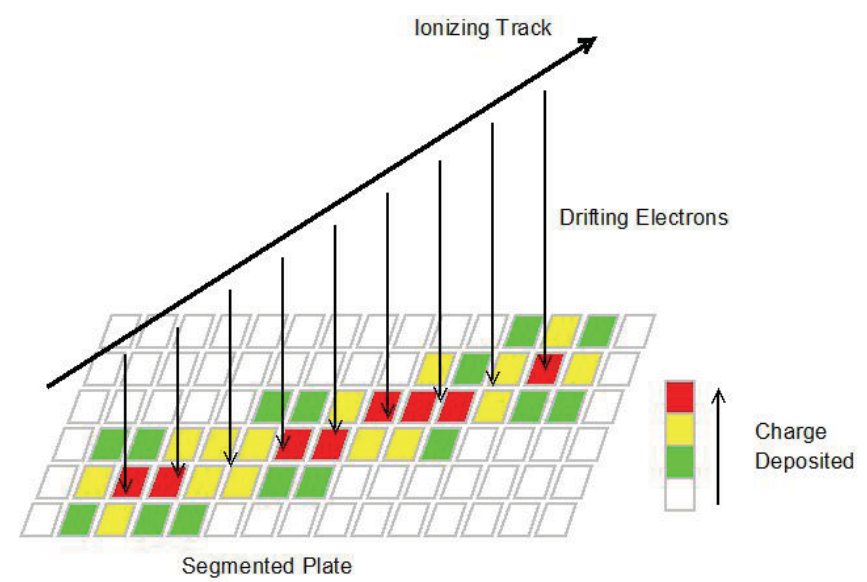

Figure 1: Illustration of the basic concept of Time Projection Chambers. 
In practice, however, building a TPC suitable for fission research is a formidable task, involving many complex systems and state-of-the-art technology. A schematic of the NIFFTE TPC is shown in Figure 2. The sample to be studied sits in the center of the cathode plane, itself located in the central plane of the electric field cage. The field cage, a printed circuit board made up of alternating strips of FR4 and gold, supplies the uniform electric field required to guide the ionization electrons towards the readout planes at the ends of the chamber. The field cage sits inside an aluminum pressure vessel, which can hold pressures of 5 bar, although typical operation pressure is approximately 980 Torr. The gas handling system, developed by Colorado School of Mines, can mix and supply up to three unique gases to the chamber. At each end of the chamber there is a readout plane ("pad plane"), made up of 2976 individual hexagonal pads. MICROMEGAS gas detectors are used to amplify and read out the drifted charge on each pad. A complete description of the fission TPC design is will be available in a NIM article to be published soon.

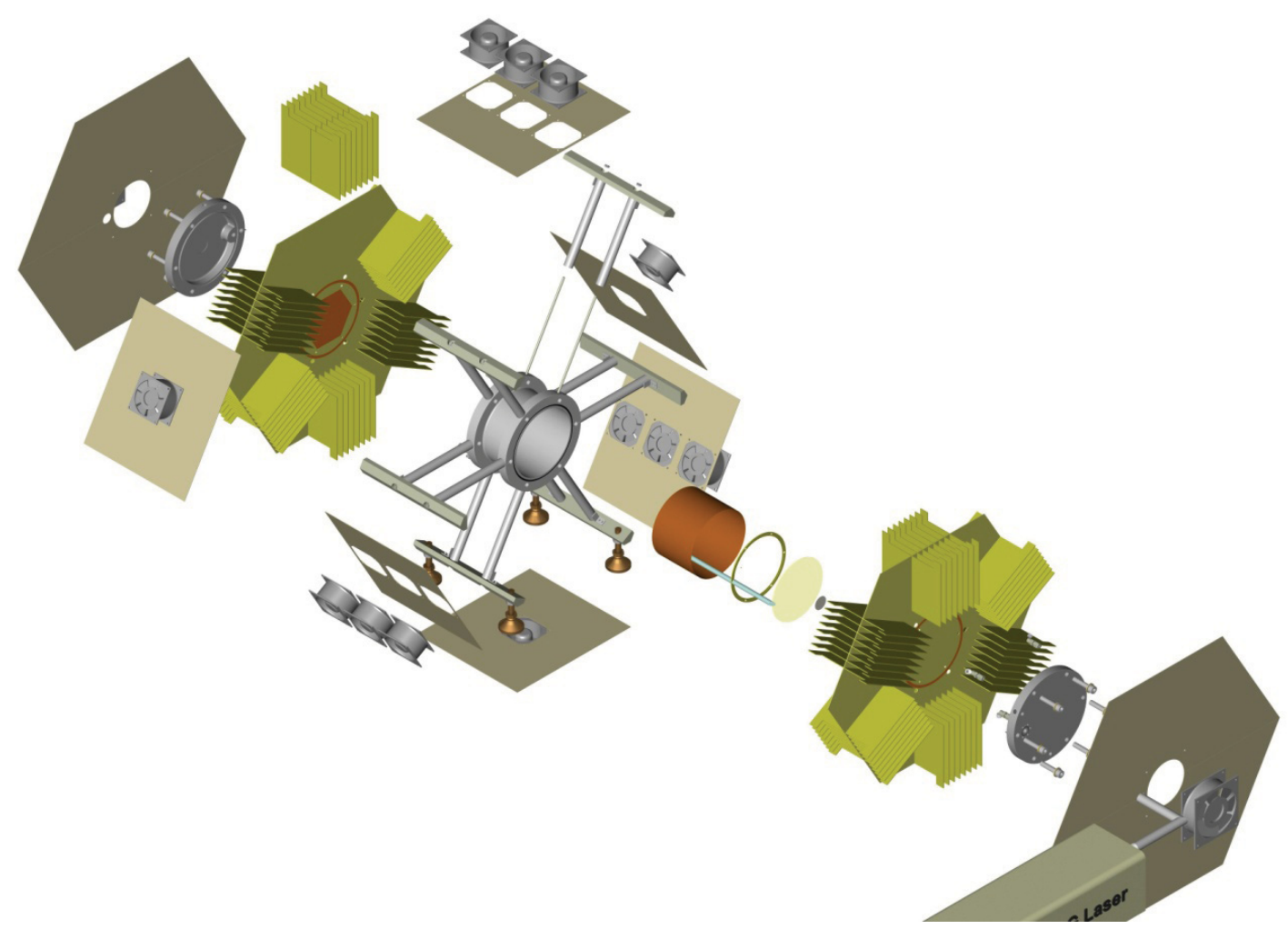

Figure 2: Expanded schematic of NIFFTE TPC. A NIM article is in preparation that fully describes the system and performance.

The 2976 pads on each readout plane are grouped into "sectors" of 28 or 32 pads/channels. Sectors consisting of 28 channels are referred to as "runt sectors". 12 sectors and 4 runt sectors are grouped into equilateral triangles called sextants - six sextants make up one pad plane. The mapping of the pad planes is shown in Figure 3. The data collected for this analysis had only one complete sextant (sextant 0 ) instrumented. 


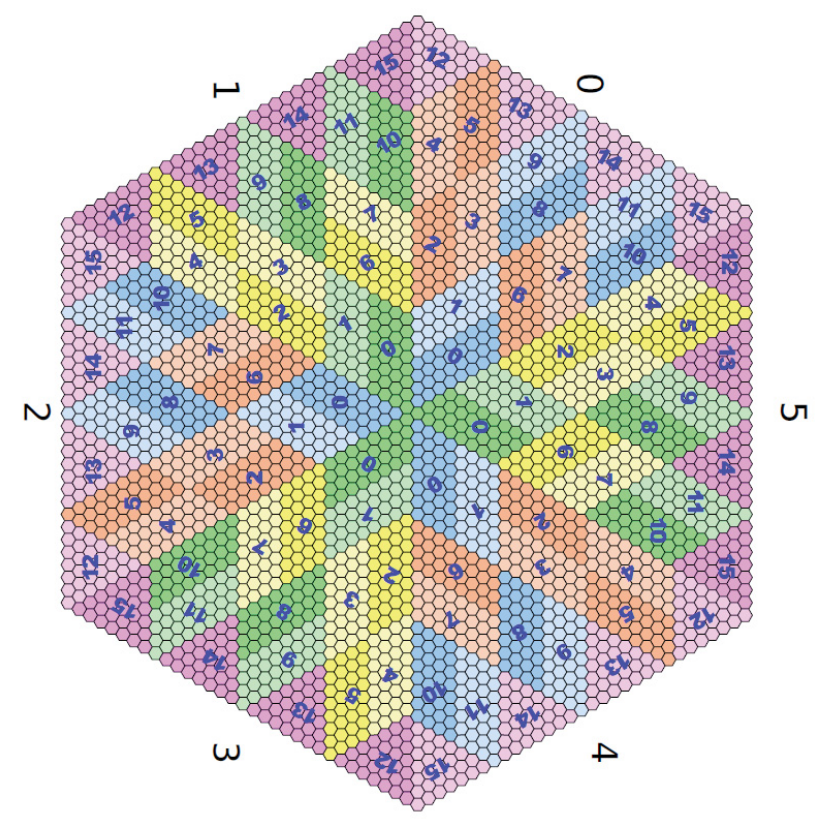

Figure 3: Pad plane mapping. The numbers on the outside refer to sextants; the numbers inside refer to sectors within the sextant.

Sectors are read out using EtherDAQ assemblies (Figure 4): 32-channel preamplifier cards connected to EtherDAQ cards equipped with analog-to-digital converters (ADCs) and field-programmable gate arrays (FPGAs). There are 16 EtherDAQ assemblies per sextant (496 channels). These assemblies digitize signals at 50,000 samples per second and send the waveforms via Ethernet to the data acquisition (DAQ) computer for further processing. Online and offline software allows for realtime viewing of the individual waveforms and reconstructed particle tracks. There is also extensive monitoring and recording of slow control information such as temperatures, gas pressure and flow, data rates, and beam status. The system is operable remotely by computer, iPad or iPhone, to relieve the burden of travel to the TPC for monitoring and adjusting.

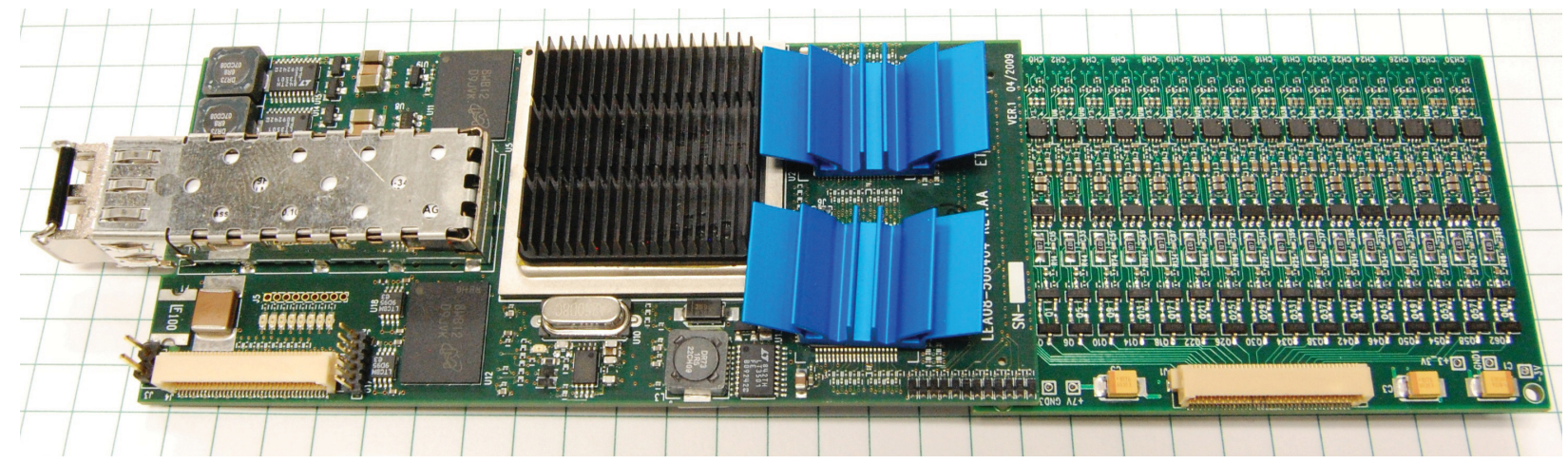

Figure 4: An EtherDAQ assembly consists of preamplifier card (right) and EtherDAQ card (left). There are 32 channels on each card and 192 cards in a fully instrumented TPC. An IEEE article is in preparation that fully describes the design and performance characteristics of this specially designed high speed, high-bandwidth system. 


\section{Los Alamos Neutron Science Center}

The experiments to be discussed in this report took place at the Los Alamos Neutron Science Center (LANSCE) facility. LANSCE is a National User Facility that provides the scientific community with intense sources of neutrons for civilian and national security research. The main feature of the facility is a linear accelerator (linac) that accelerates both positive and negative hydrogen ions to $800 \mathrm{MeV}$. The positive ion beam is used for isotope production. The negative ions are directed into tungsten spallation targets to drive two neutron beam facilities: the Manuel Lujan Jr. Neutron Scattering Center, and the Weapons Neutron Research (WNR) facility.

Much of the scientific research performed at the Lujan Center focuses on materials science, although there are three flight paths devoted to nuclear science research. The moderated spallation target produces beams with neutron energies ranging from sub-thermal to approximately $500 \mathrm{keV}$. These beams complement those produced at the WNR facility, which uses a bare (unmoderated) tungsten spallation target to produce a high-energy "white" neutron spectrum with energies ranging from $100 \mathrm{keV}$ to $600 \mathrm{MeV}$.

A schematic depicting the LANSCE beams is shown in Figure 5. The TPC experiments take place on the 4FP90L flight path at the WNR facility.

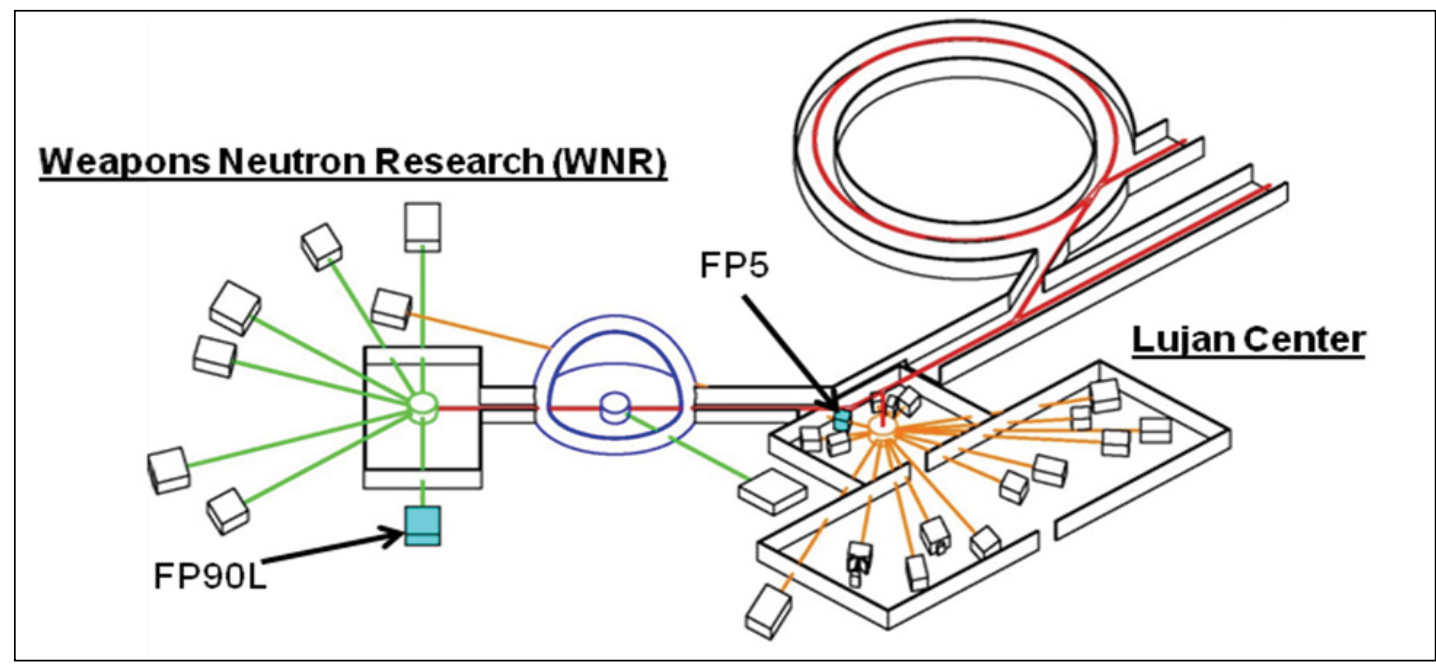

Figure 5: The Los Alamos Neutron Science Center. The TPC is situated on a dedicated beam line at the WNR, referred to as FP90L.

\section{Weapons Neutron Research Facility}

The Weapons Neutron Research (WNR) facility is at present the most intense highenergy neutron source in the world, and has six flight paths available for a variety of nuclear science measurements. Each flight path views the spallation target from a different angle (relative to the proton beam), which results in a different neutron energy spectrum. The most forward angle flight paths have higher neutron intensities 
at high energies; more backward angle flight paths have significantly lower intensity at high energies but higher intensities of low-energy neutrons. The incident neutron energy is typically determined using the time-of-flight technique.

The radiofrequency of the linac determines the time structure of the neutron beams at WNR. Typically, the time structure is as depicted in Figure 6. Macropulses of $625 \mathrm{~ns}$ duration (the time the linac radiofrequency driver is on) arrive at the spallation source at a frequency of $40 \mathrm{~Hz}$. Within each macropulse are sharp micropulses separated by 1.8 us. This micropulse spacing is set such that slower neutrons in the pulse can reach the detector before the higher energy neutrons from the next pulse arrive, but can be adjusted if longer spacing is needed.

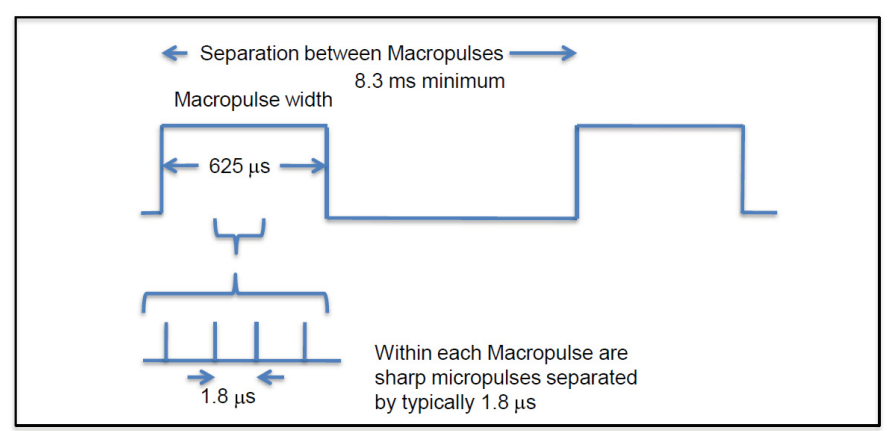

Figure 6: Time structure of proton beam for typical WNR operation. Source: LANSCE Nuclear Science Research Facility User Guide.

\section{The 4FP90L Flight Path}

The 4FP90L flight path has been dedicated to TPC operations since 2010. The flight path views the spallation target from a 90 degree angle relative to the incident proton beam. One of the more backward-angle flight paths, 4FP90L is ideal for fission experiments as the average flux (in the hundreds of MeV range) is lower than that of the more forward flight paths. A relatively short flight path, 4FP90L sits directly against the spallation target bulk shielding. The beam pipe is evacuated to the shutter, and then the beam continues in air to the detector. The measured and simulated neutron flux on the 4FP90L flight path is shown in Figure 7, for a nominal detector position of $10 \mathrm{~m}$ from the neutron production target.

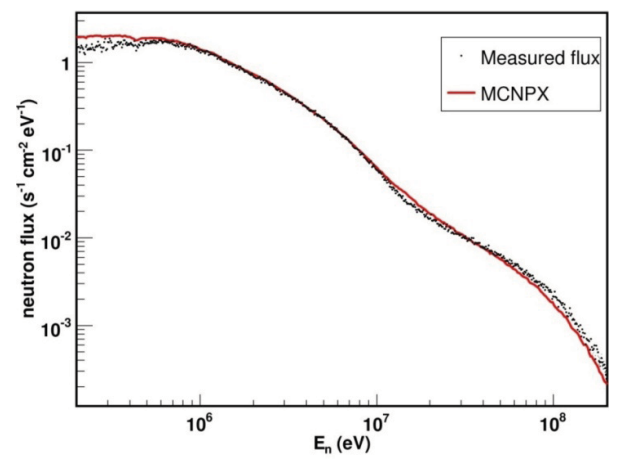

Figure 7: Measured and simulated flux at the 4FP90L flight path. 


\section{Experiment}

There were two TPC engineering experiments involving mixed-isotope (U235 and U238) samples during the 2011-2012 LANSCE Run Cycle. Both experiments took place on the 4FP90L flight path at the WNR facility. Using a combination of in-shutter and in-room collimation, a circular neutron beam $1 \mathrm{~cm}$ diameter (Figure 8 ) is delivered to the TPC target. The TPC was positioned approximately $8 \mathrm{~m}$ from the spallation target.

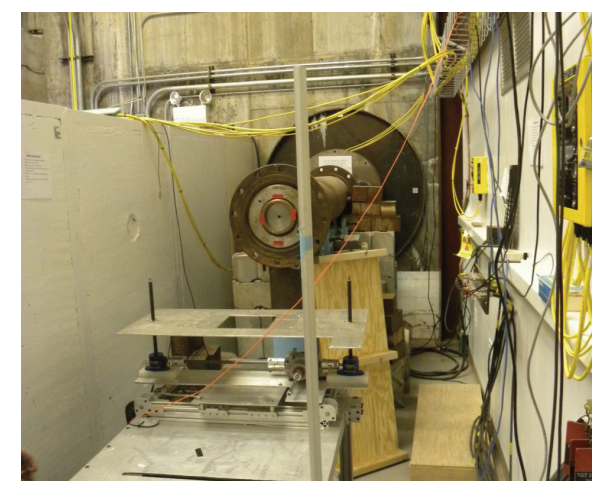

Figure 8: Collimation used in 2011-2012 LANSCE Run Cycle on 4FP90L. The in-room collimation can be seen in the background protruding from the shield wall. In the foreground is the TPC aluminum stand that supports the detector and houses much of the necessary support electronics.

The TPC was equipped with 16 EtherDAQ assemblies, and data were collected with one sextant (496 channels). Notably, the required shifts during these experiments were covered by external collaborators at their home institutions due to online software developed at Abilene Christian University. For the first time in this project, it was possible to monitor and control all relevant parameters remotely by computer, iPad or iPhone, limiting on-call intervention and added travel.

New hardware and slow controls implemented prior to the 2011-2012 LANSCE run cycle included: a new pressure vessel, pad plane, bus boards (power assemblies for EtherDAQ cards), high voltage circuitry, high voltage power supplies, and temperature and pressure monitors. The details of these upgrades are documented in previous quarterly reports.

\section{Run1：U238+U235 - November 29 - December 17, 2011}

During the November accelerator maintenance outage, a mixed-isotope sample $\left(123.6 \mu \mathrm{g} / \mathrm{cm}^{2} \mathrm{U}-235\right.$ and $218.5 \mu \mathrm{g} / \mathrm{cm}^{2} \mathrm{U}-238$ ) on a thick aluminum backing was installed into the TPC (Figure 9). Initially, the sample was placed with the deposits facing the inactive pad plane and background data on neutron interactions with the aluminum backing were taken for approximately 7 days. The sample was then flipped such that the actinide deposits faced the active pad plane and the gap between the deposited samples was centered (by eye) in the center of the active sextant (Figure 10). 


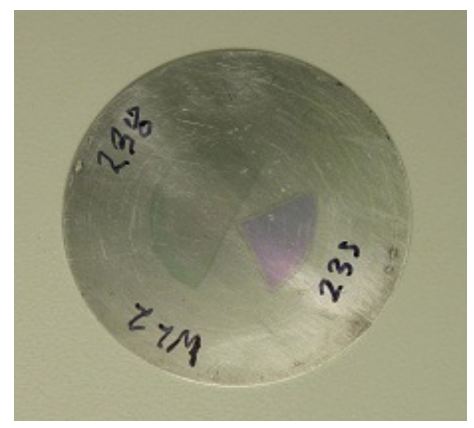

Figure 9: Mixed isotope sample used during the first U235-U238 measurement of the 2011-2012 LANSCE Run Cycle was fabricated at Oregon State University.

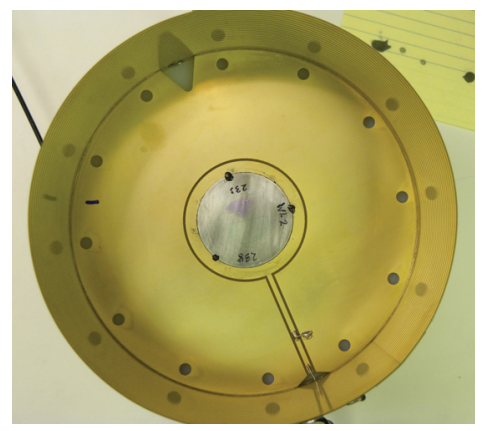

Figure 10: Mixed isotope sample from Figure 9, inserted into the field cage. The blue line on the outside edge indicates the center of the active sextant.

Due to construction of a new building at WNR, data collection was limited to off hours to minimize radiation risks to workers in the area. Additionally, frequent trips of the high voltage system resulted in 18 channels dying during the run. Other minor learning experiences that resulted in a loss of potential data-collection included: improper card cabling; a failure of the gas handling system programming controls; two DAQ software failures; and time when the accelerator beam was down. Despite these issues, data were collected for approximately $72 \%$ of the available beam time. A breakdown of how the available time was allocated is shown in Figure 11.

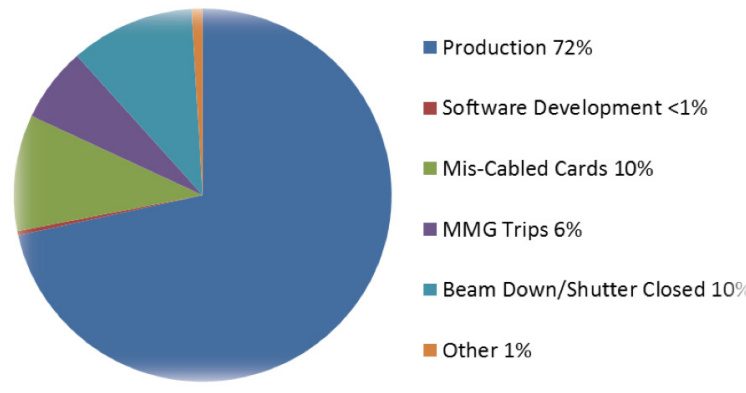

Figure 11: Beam time allocation, first mixed isotope run of the 2011-2012 LANSCE run cycle. 


\section{Run2 : U238+U235 - January 12 through 27, 2012}

During the January accelerator maintenance outage, a second mixed-isotope sample $\left(158.8 \mu \mathrm{g} / \mathrm{cm}^{2} \mathrm{U}-235\right.$ and $\left.342.8 \mu \mathrm{g} / \mathrm{cm}^{2} \mathrm{U}-238\right)$ on a thick aluminum backing was installed into the TPC (Figure 12). The sample was oriented such that the actinide deposits faced the active pad plane and the gap between the samples was centered (by eye) in the center of the active sextant.

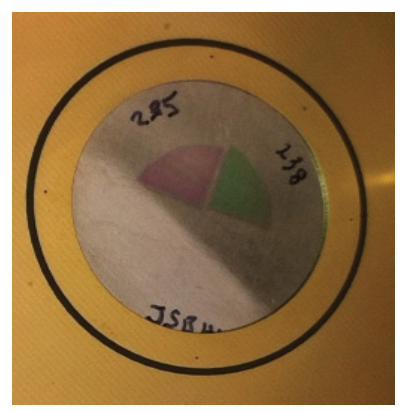

Figure 12: Second mixed isotope sample used in the 2011-2012 LANSCE run cycle was fabricated at Oregon State University.

New hardware implemented in this run included a Power and Clock Distribution Unit (PCDU) for low voltage control, and a special filter circuit to reduce the damage in system trips. The system trips are under investigation and will most likely be eliminated. As a failsafe plan, new electronics have been developed to withstand the events that result in electronic failure, insuring that the number of dead channels is minimized. Software and slow control improvements included remote control of all power (high voltage and low voltage) and new monitors for gas flow, beam intensity, high voltages, and improvements to online monitoring.

WNR construction was paused during this period and allowed for 24-7 data collection. As expected during detector development periods, there were a few complications during these runs. Specifically, down time accrued due to several system trips; crash of the gas handling system programming controls; one failure of the DAQ software, and accelerator service periods. However, as with the previous run, a significant portion of time available (69\%) was spent collecting quality data. A breakdown of how the available time was allocated is shown in Figure 13.
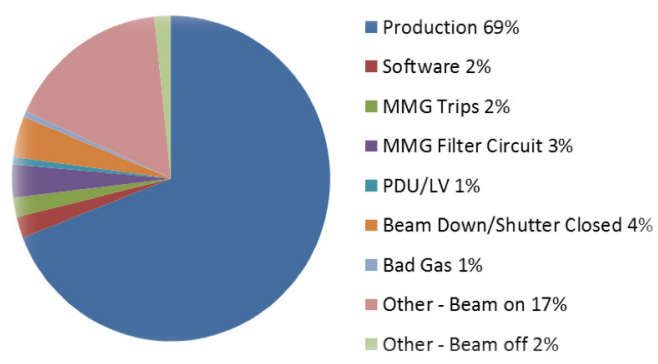

Figure 13: Beam allocation, second mixed-isotope run of the 2011-2012 LANSCE run cycle. 


\section{Analysis}

One of the revolutionary aspects of the NIFFTE TPC, compared to the ionization chambers used in previous measurement, is its ability to perform complete threedimensional particle tracking. This capability is necessary to remove the systematic uncertainty caused by mis-identification of fission fragments and alpha particles in all previous fission cross section measurements. This analysis is focused on the improvements in alpha/fragment particle discrimination that the TPC was designed to maximize. The software development effort for this project is being led by CalPoly at San Luis Obispo with contributions coming from across the collaboration.

There are several noteworthy limitations with these data sets. Firstly, the limited angular coverage (single-sextant) and the extended target complicates the analysis as many of the tracks in these data are only partially reconstructed in the limited instrumented volume. This impacts the achievable particle identification separation resolution as a whole, as partial tracks are indistinguishable from low-energy, short, complete tracks. However, a subset of tracks are analyzed that demonstrate the power of the TPC method and some results from a half instrumented TPC are included for completeness. More practically, trips in the system described earlier damaged a large portion of the central read-channels of the pad plane, which resulted in an even greater number of partial tracks with uncertain vertex information. Finally, the lack of high-precision timing information in this dataset excludes the use of timeof-flight methods that are traditionally used to distinguish U-235 and U-238 events. Taking these issues into consideration, along with the knowledge that U-238/U-235 data are currently being collected at LASNSCE with a full pad plane and timing information, the decision was made to use the single-sextant data to investigate the effect of particle tracking on alpha/fragment particle discrimination and to tune analysis procedures for the improved data to come.

For each of the mixed isotope samples described above, the following criteria were examined:

- Track reconstruction:

- Track vertexing: Can one use tracking alone to distinguish U-235 and U-238 sample deposits?

- Angle reconstruction: What are the effects of limited angular coverage on track azimuthal and polar angles?

- Alpha particle/Fission fragment discrimination:

- Track length vs. energy deposition: What improvements does tracking information bring to particle discrimination?

All references to particle tracking imply the following procedure: particle track candidates are identified event by event using a Hough transform of the TPC data. The Hough particle track candidates are then fit using a Kalman filter to estimate track parameters and errors, including start point, end point, azimuth and polar angle, and total energy deposited along the track. These track finding/fitting procedures are well established in the high-energy physics community and have been specifically adapted for use with this specific TPC. 


\section{Track Reconstruction}

An attempt was made to determine how much information is gained from particle tracking alone, without refined calibration constants or high precision timing. The investigation focused on two aspects: reconstruction of track start positions (vertices) and angles, both as a function of deposited charge. For illustrative purposes, a simple schematic of the detector geometry is shown in Figure 14. Using the coordinate system provided, one can see that the incident beam direction is negative- $z$, and the active sextant encompasses an area of positive-y and negative-x. In terms of polar $(\Theta)$ and azimuth $(\Phi)$ angles, this sextant includes $0<\Theta<90$ degrees, and $90<\Phi<$ 150 degrees.

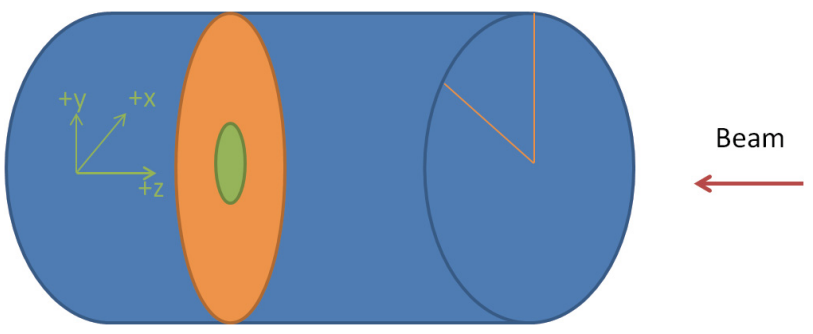

Figure 14: Simple schematic of detector geometry and coordinate system. The instrumented portion is shown as the pie shape at the right side of the TPC cylinder. This figure is only illustrative. The target plane in the actual TPC system is centered in the active volume.

\section{Vertexing}

Reconstructed track vertices are plotted in Figure 15-18 below. To guide the eye, the black line in each figure indicates the center of the sextant, and the red lines are 0.5 $\mathrm{cm}$ offset from the center. The three rows in each figure indicate selections of the total ADC of the particle track, an uncalibrated measure of the energy deposited in the detector. The top row includes all events, the middle row includes only events with total ADC less than 2000 (primarily low-charge particles such as alpha particles and protons), and the bottom row includes only events with total ADC greater than 2000 (primarily fission fragments, although some higher-energy alpha particles could have total ADC within this region). As expected, the events with high ADC deposition have track starting positions focused at the center of the sextant (where the actinide samples are located), and the bulk of the track vertices low-ADC events are distributed throughout the sextant (confirming that these are due to reactions other than those of interest).

In Figure 15 and Figure 17, the leftmost column includes all events, and the rightmost column includes events with track angles within the active sextant (see Figure 14 and surrounding text). Clearly, limiting the data to tracks within the active sextant improves the vertex resolution. In Figure 16 and Figure 18, angular cuts have been implemented in all plots, and a crude U-238/U-235 separation is attempted. Assuming that the sample was perfectly centered, the U-238 deposit should be below the center line and the U-235 sample should be above the center line. The $0.5 \mathrm{~cm}$ offset is included to mitigate effects of an off-centered sample. For future analyses, when high precision timing (neutron energy) information is included in the data stream, it should be possible to optimize these sample boundaries on mixed-isotope targets using the fission cross-section threshold. 


\section{Track Vertices - Run1 : U238+U235}

The data set used for this analysis included 20 hours of data, 10 runs of 2 hour duration. It was selected based on stability criteria - through the entire 20 hours, there were no significant technical issues or beam outages.

The sample employed in this run consisted of a semi-circle of U-238 and a wedge of U235 (see Figure 9), each $0.5 \mathrm{~cm}$ in diameter. The actinide samples were deposited on a thick aluminum backing. In addition to tracks from alpha particles and fragments produced in neutron-induced fission, tracks from reactions induced on gas particles, the aluminum backing, and detector materials were also observed.
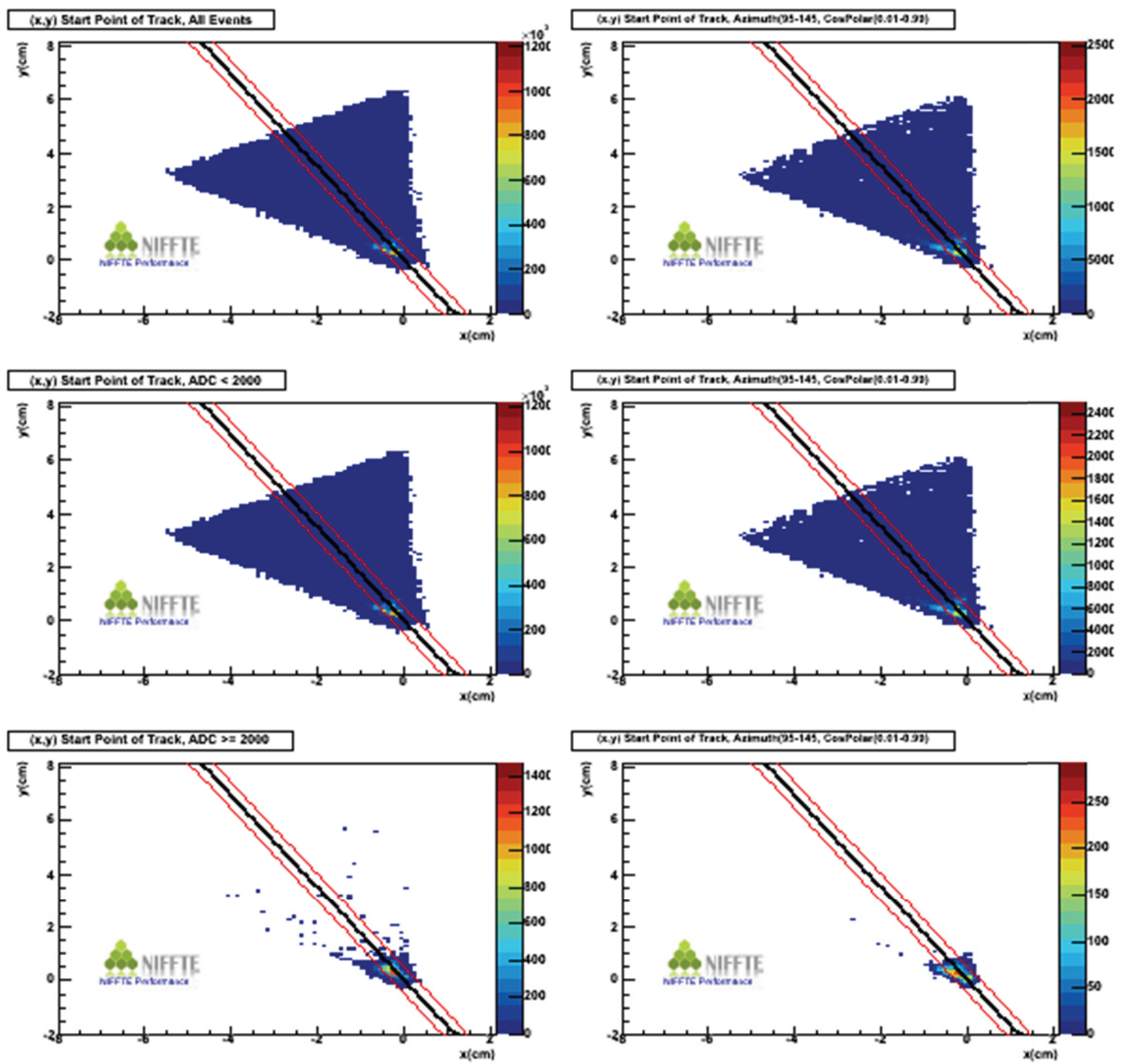

Figure 15: Track start positions, with cuts on track angle and ADC (see text). 

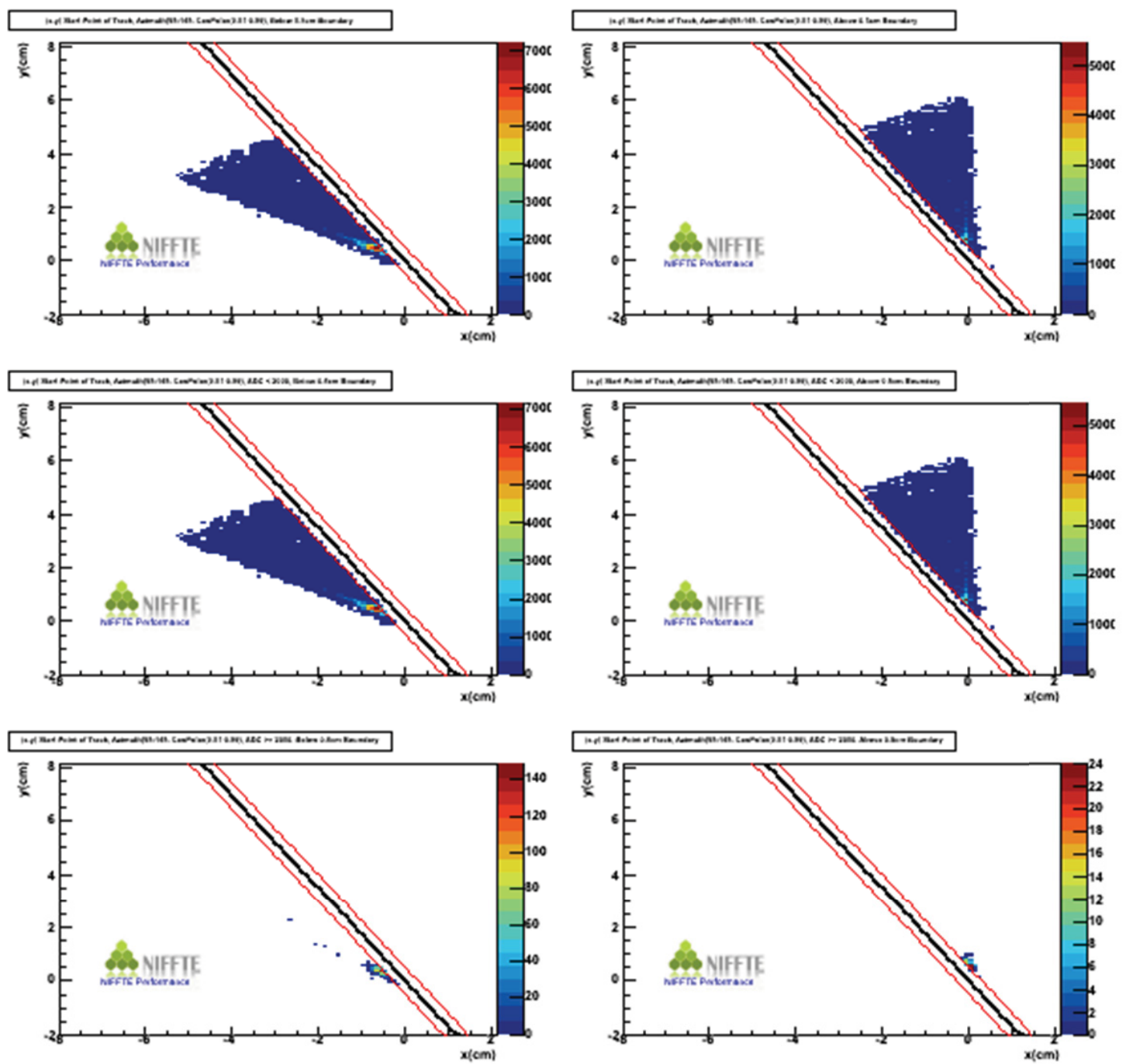

Figure 16: Track start positions, with cuts on track angle, ADC, and start position (see text).

\section{Track Vertices - Run2 : U238+U235}

The data set used for this analysis included 20 hours of data, 10 runs of 1 hour duration. It was selected based on stability criteria - through the entire 20 hours, there were no significant technical issues or beam outages.

The sample employed in this run consisted of one quarter-circle of U-238 and another of U235 (see Figure 10), each $0.5 \mathrm{~cm}$ in diameter. The actinide samples were deposited on a thick aluminum backing. In addition to tracks from alpha particles and fragments produced in neutron-induced fission, tracks from reactions induced on gas particles, the aluminum backing, and detector materials were also observed. 

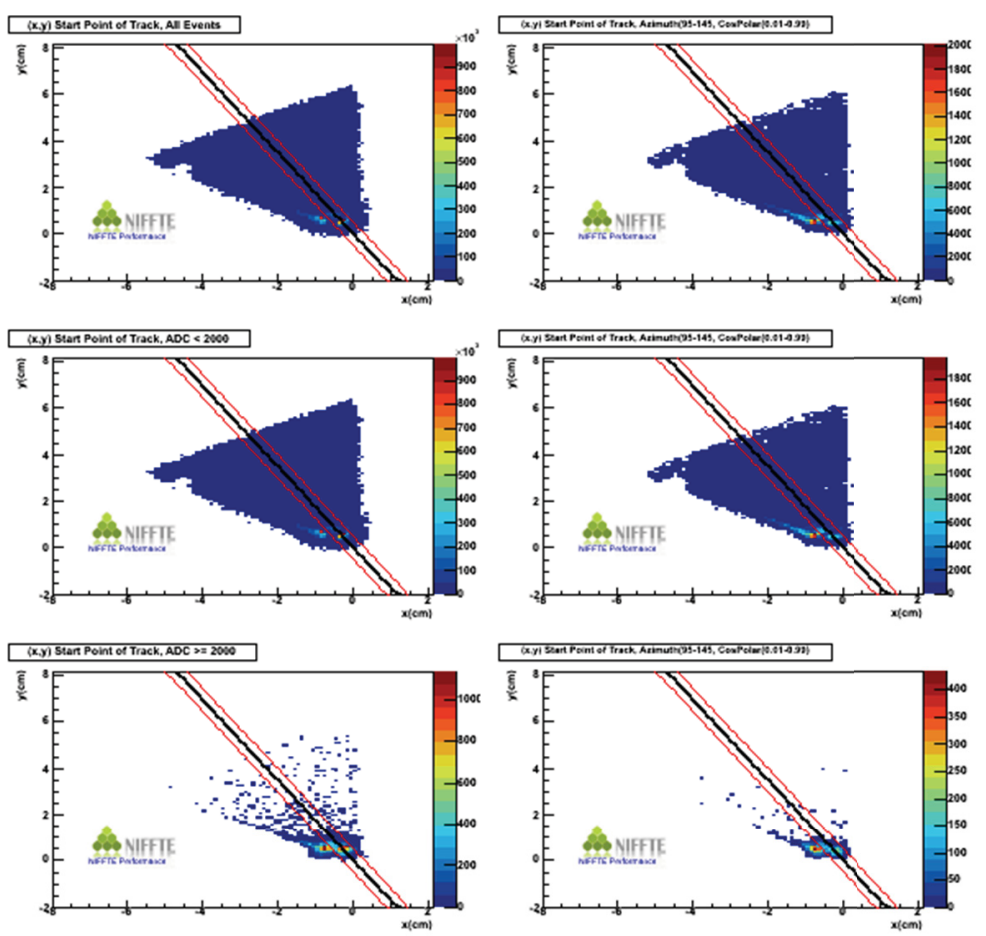

Figure 17: Track start positions, with cuts on track angle and ADC (see text).
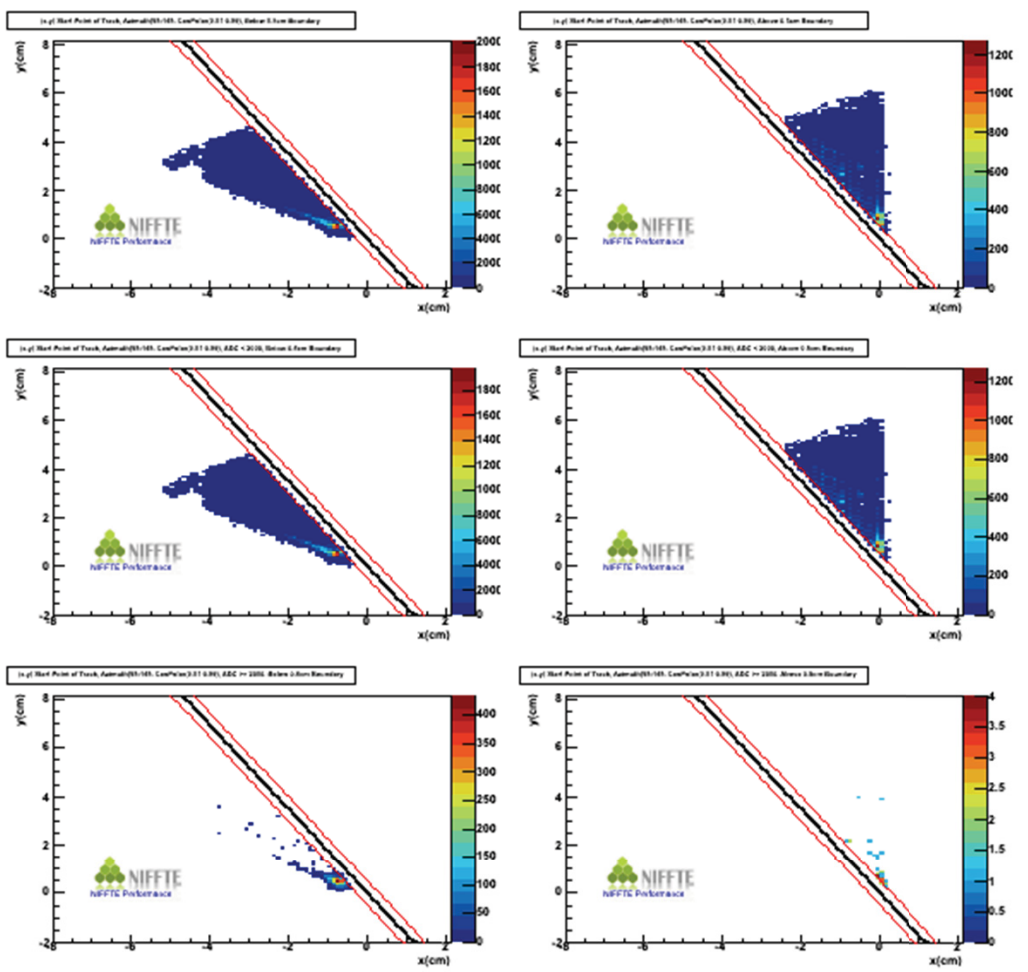

Figure 18: Track start positions, with cuts on track angle, $\mathrm{ADC}<$ and start position (see text). 
By closely examining Figure 15 and Figure 17, one observes significantly worse resolution in the latter data set. This is due to a large number of dead channels, highlighted in white in Figure 19. The lack of information from these channels makes it difficult to accurately reconstruct track vertices, and efforts are underway to reduce the number of damage pixels and develop algorithms to correct for this effect.

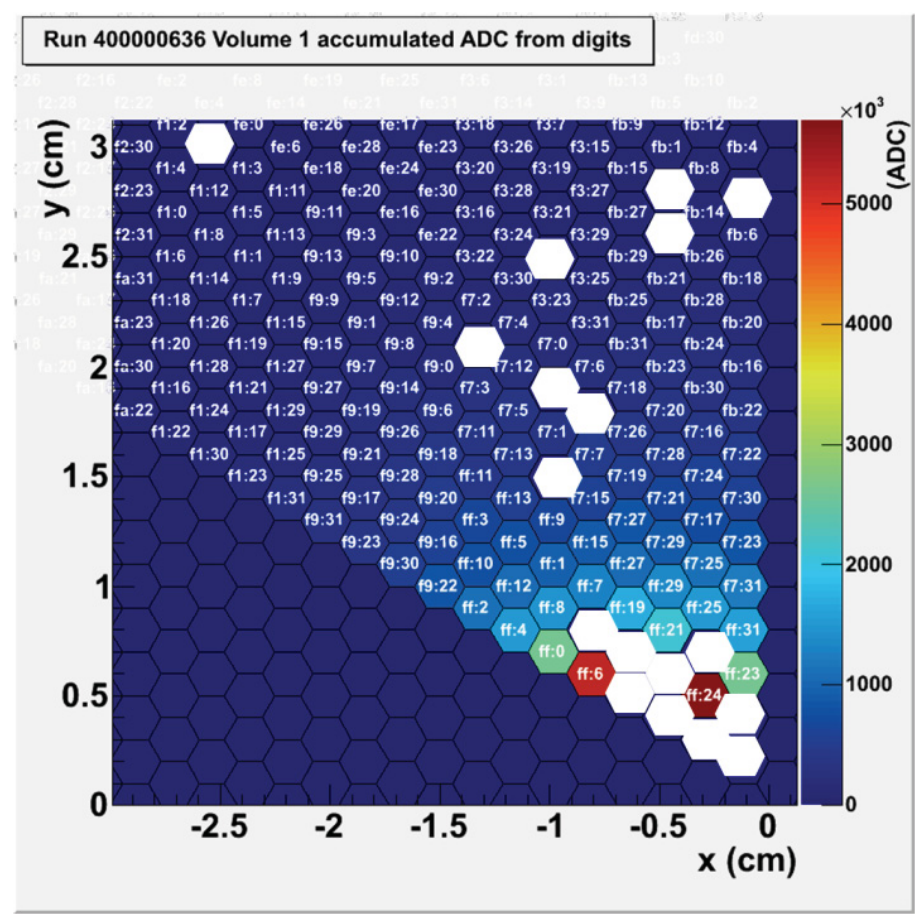

Figure 19: Accumulated (total) ADC deposited per pixel, first run of second data set. Dead pixels are highlighted in white. Throughout the 20 -hour data collection period, 8 more channels were lost (in addition to those shown here). The cause of death is under investigation and is believed to be a result of unexpected noise in the grounds of these very sensitive electronic circuits.

\section{Track Vertices - Full-sided TPC U238+U235}

The data set used for the following plots is from beam running at the LANSCE facility with a specially prepared target where a semi-circle of U235 is in the top position and a U238 semicircular target is below it. In this configuration of the TPC, where an entire side is instrumented, artifacts due to limited viewing are completely eliminated and included here for an up to date comparison with the engineering sextant data. The two isotopes are clearly visible, where the difference in statistics are driven by the total cross section difference between the two, folded with the energy spectrum of the neutron beam (see Figure 20). This type of information has never been available before in fission cross measurements and demonstrates the basic pointing capabilities of the system, which when fully developed will be used to assay the targets and unfold the non-uniformities of the beam and the samples. 


\section{Track X-Y Plane Vertex Fission Fragments}

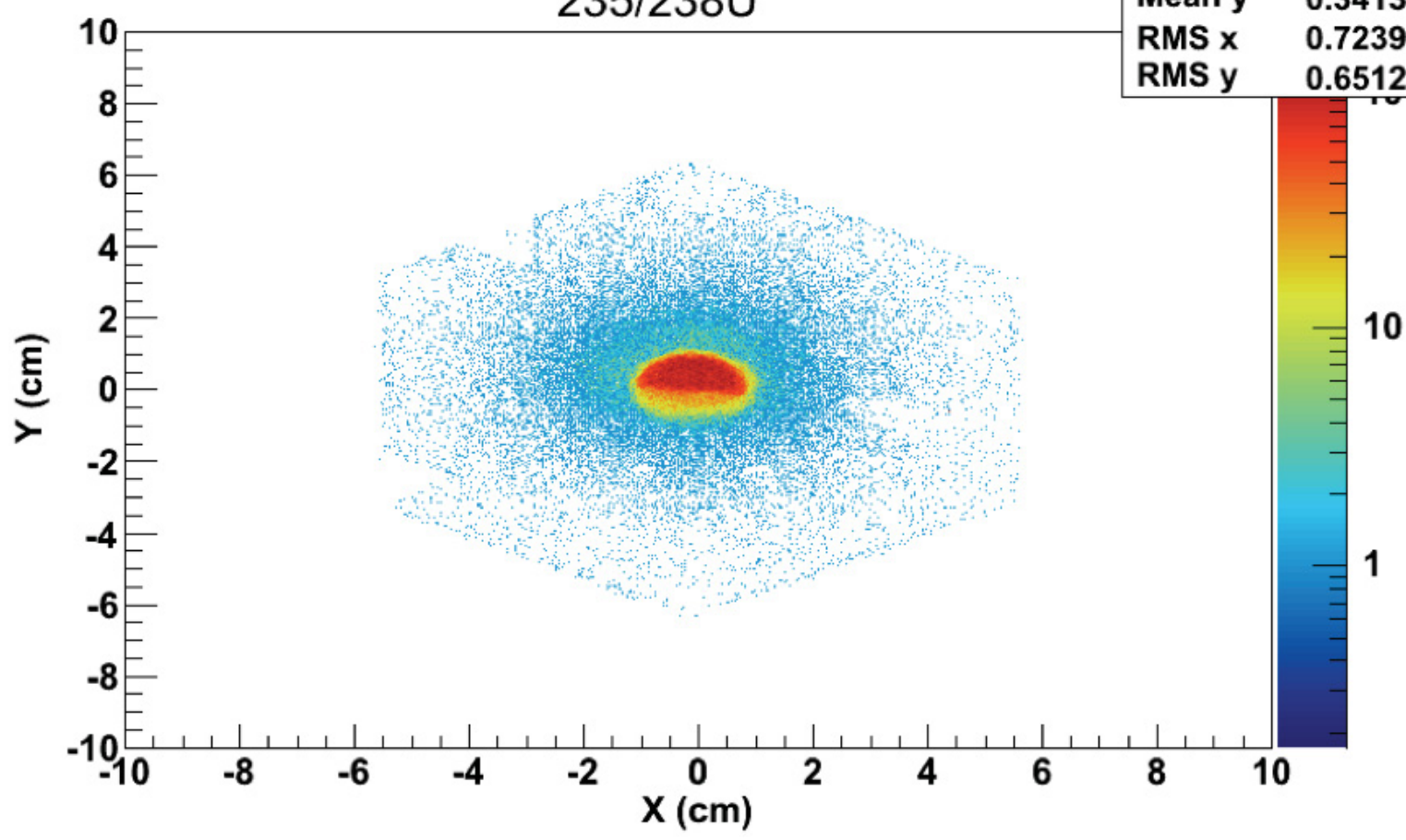

Figure 20: Shown here is data collected using a fully instrumented side of the TPC where a high collected charge requirement is imposed to reduce the lighter particle backgrounds. The target at the center is circular, with the upper half being $U 235$ and the lower $U 238$. The statics reflect the difference in fission cross sections between the two isotopes in the energy spectrum of the LANSCE beam and are clearly separated.

\section{Angle Reconstruction}

Traditionally, one uses the azimuth and polar angular distributions to confirm the suitability of the data set - both should be flat as fission is expected to be approximately isotropic. Figure 21-24 show these distributions for the current data sets, but one should note the distributions are largely skewed by the limited angular coverage of the active area of the TPC and geometric effects at the sextant boundaries. With extensive efforts, corrections can be made for these effects, but data have been collected with a full pad plane (thereby eliminating these issues) and are also shown below.

As with the vertex plots, the three rows in Figure 21 and Figure 23 indicate selections of the total ADC of the particle track. The top row includes all events, the middle row includes only events with total ADC less than 2000 (primarily low-charge particles such as alpha particles and protons), and the bottom row includes only events with total ADC greater than 2000 (primarily fission fragments, although some higherenergy alpha particles could have total ADC within this region). The left column in Figure 21 and Figure 23 show azimuth angle distributions, and the right column show 
the polar angle distributions, plotted as cosine of this angle. Figure 21 and Figure 23 have no angular cuts applied.

Figure 22 and Figure 24 have angular cuts applied that reflect the geometry of the detector. The first three rows show the cosine polar distribution, with the all-ADC, lowADC, and high-ADC cuts. These three plots have also been restricted to azimuthal angles within the active area of the detector. The fourth row in Figure 22 and Figure 24 shows the azimuth angle distribution for all events with polar angles within the active area of the detector.

Physics conclusions should not be drawn from Figure 21-24 - these are merely included to illustrate that the capabilities to quickly plot track angle distributions has been implemented and will be used for the analysis of future data.

Track Angles - Run1 : U238+U235
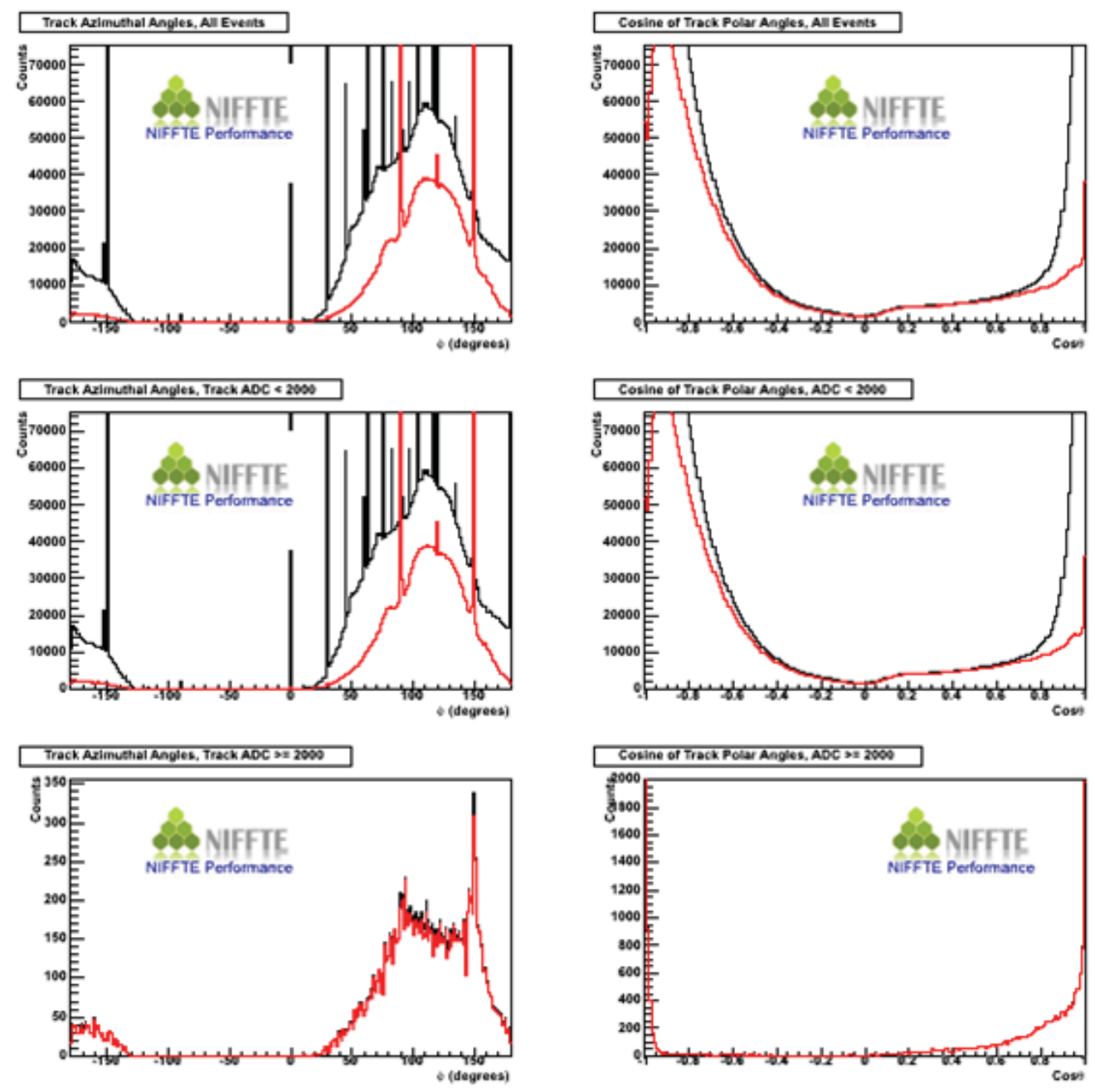

Figure 21: Track azimuth and polar angle distributions. The right column shows the polar distributions and the left column show azimuthal where no cuts to the data have been applied. 

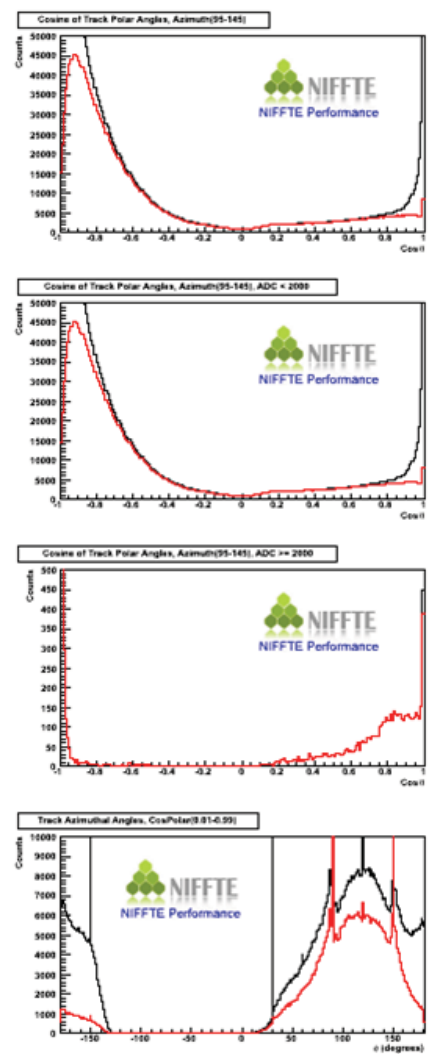

Figure 22: Track azimuth and polar distributions, with angular cuts applied.

Track Angles - Run2 : U238+U235
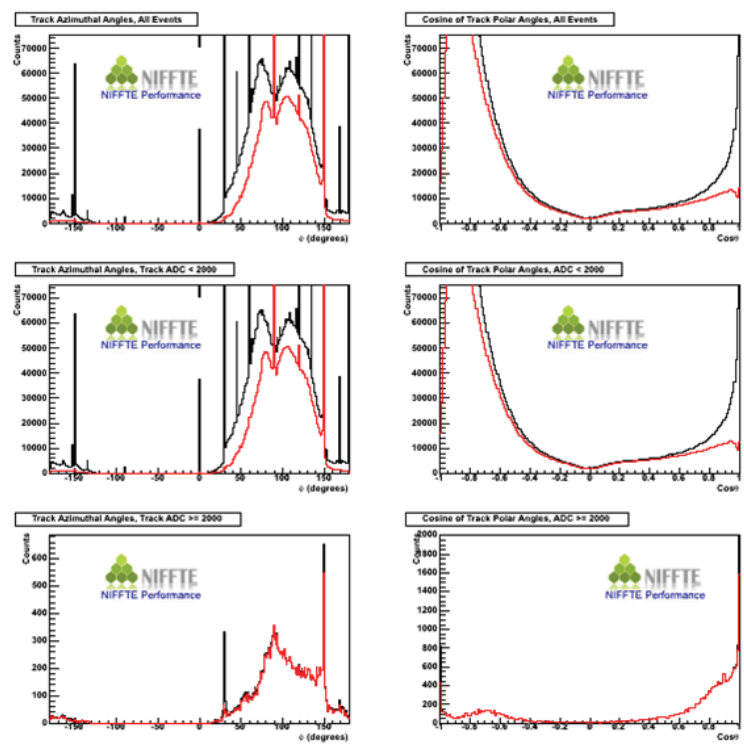

Figure 23: Track azimuth and polar angular distributions. The right column shows the polar distributions and the left column show azimuthal where no cuts have been applied. 

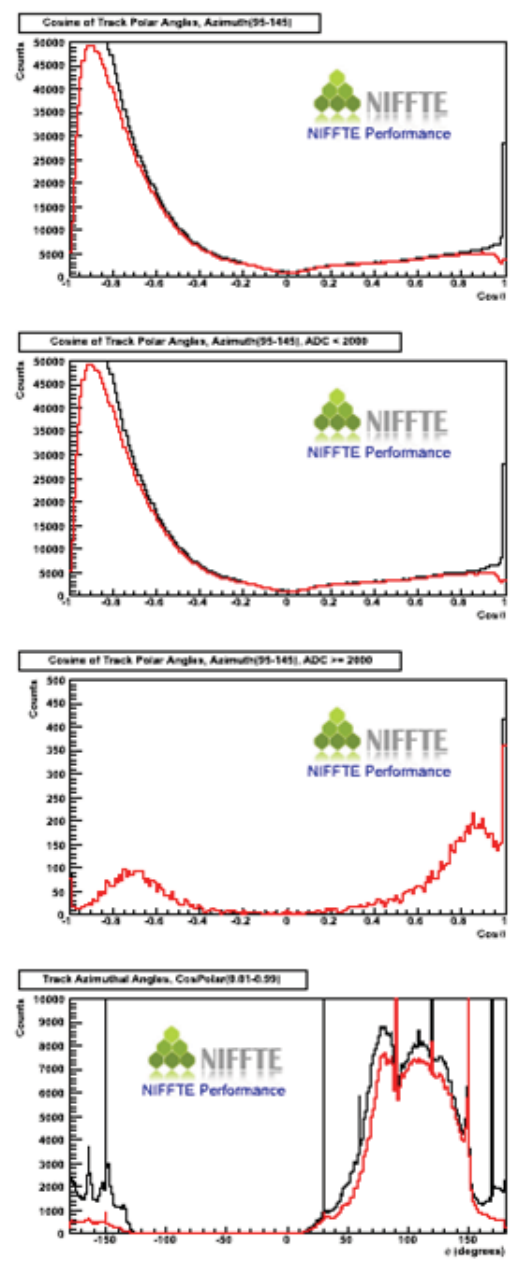

Figure 24: Track azimuth and polar angular distributions, with angular cuts applied (see text).

Track Angles - Full Side TPC U238+U235
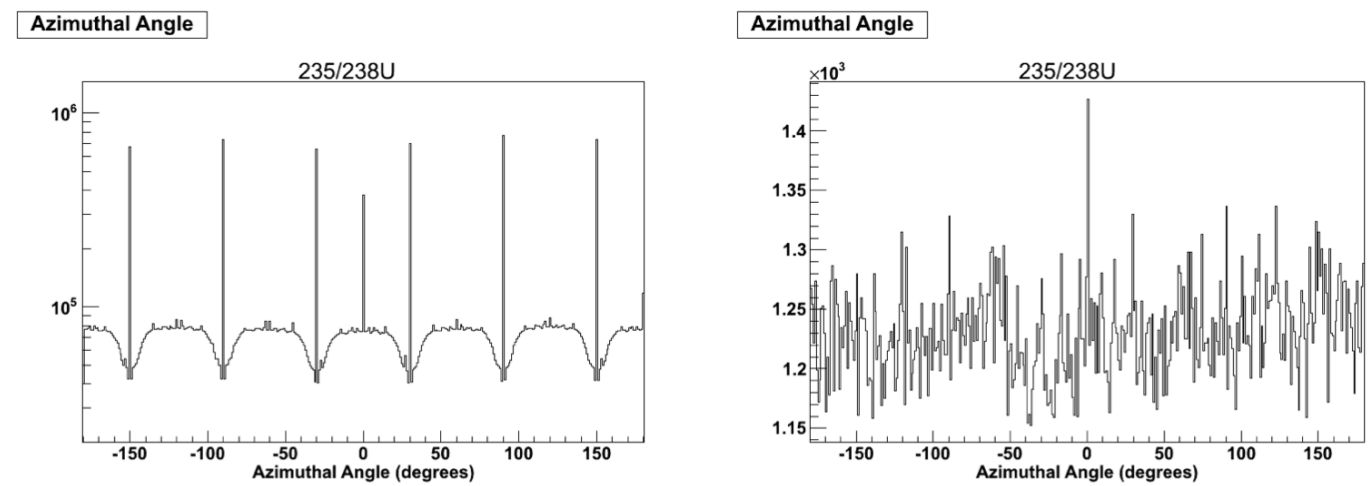

Figure 25: Here is the azimuthal angle for all tracks for a fully instrumented pad plane (left) and for fission products (right). The six-fold pattern in the left plot is a result of the hexagonal geometry and the behavior of the track fitter for some of the noise in the data. The right plot is a subset of events that appear by total charge to be fission products and here you can see the distribution is flat. 


\section{Alpha/Fragment discrimination}

As alluded to previously, the primary purpose of this analysis was to determine whether including tracking information could help with a main source of systematic error in previous fission cross-section measurements: the ability to distinguish alpha particles from fission fragments.

For both data sets, we examined at the total ADC deposited in the detector, with: (1) no angular limitation applied; (2) angles limited to those within the active sextant of the detector; (3) "forward" angles, meaning polar angles between 0 and 45 degrees, where 0 degrees is parallel to the beam axis; and (4) "backward" angles, meaning polar angles between 45 and 90 degrees, where 90 degrees is parallel to the cathode plane. These plots are shown in Figure 26 and Figure 31. The columns, from right to left, correspond to (1)-(4) above. The rows correspond to total ADC, total ADC plotted as a function of the (cosine of) polar angle, and the track length vs the total ADC.

Noteworthy features of Figure 26 and Figure 31 include:

- With regard to total $A D C$, one can see improved separation between protons (ADC less than 500) and alpha particles (ADC between 500 and 2000) as angular cuts are applied, and larger relative contributions of alpha particles (compared to high-ADC fission fragments) at backward track angles.

- With regard to track length vs. total ADC, one observes two distinct bands for low-energy/light particles (protons, alphas, etc.) and fission fragments. The lighter charged particles travel further through the detector but deposit less energy, and the fission fragments have shorter, higher-energy track. One also notes improved separation between alpha particles and fission fragments at backwards angles.

Next, as a way to compare the TPC data to fission chambers, we used the total ADC information to determine where a one-dimensional cut between alpha particles and fission fragments would likely be made. To find the cut position, we fit the alpha particle peak in the total ADC spectrum and chose the separation point based on where the Gaussian fit function would correspond to less than one alpha particle. The fits and corresponding one-dimensional cut locations are shown in Figure 27 and Figure 32. Columns correspond to (1) active sextant angular cuts; (2) forward angles; and (3) backward angles. Rows correspond to the total ADC spectrum and the twodimensional track length vs. total ADC plot. It is clearly visible when examining the two-dimensional track length vs. total ADC plot that the cuts based on total ADC information alone would not sufficiently discriminate between alpha particles and fission fragments. There are many medium-length $(5-7 \mathrm{~cm})$ alpha particle tracks that deposit sufficient energy to be included in data based on this one-dimensional cut.

Figure 28 and Figure 33 demonstrate the effectiveness of a two-dimensional cut on track length vs. total ADC at discriminating fission fragments from lighter particles. As before, columns represent sextant, forward, and backward angular cuts. By eye, a polygonal cut was made based around the fission fragment band in the twodimensional plot (row 1, Figure 28 and Figure 33). This cut was then applied to the total ADC spectrum and the results are shown in row 2 of Figure 28 and Figure 33. Looking at the low energy limit of the total ADC histogram one can see the protons and many of the alpha contributions have been eliminated. This crude cut could likely be optimized further, providing even better separation capabilities. 
Figure 29 and Figure 34 compare the one-dimensional cut based on total ADC alone (top row) and the two-dimensional cut based on track length and total ADC information (bottom row). Columns correspond to sextant, forward, and backward angular cuts. Clearly, seepage of the high-energy tail of the lighter particles is greatly reduced when a two-dimensional cut is applied.

Finally, an attempt was made to understand which kinds of particles contribute to the border region between the lighter particles and fission fragments in the track length vs. total ADC plot. Figure 30 and Figure 35 show representative tracks from (left) the alpha band, (center) the overlap region of the two-dimensional cut, and (right) fission fragments. It appears these particles in the overlap region may be partial tracks, due to limited angular coverage and dead channels - therefore the separation between alpha particles and fission fragments should be greatly improved once (1) the full pad plane is instrumented and (2) the number of dead channels is reduced.

The plots at the end of the section also include data from a separate run from a U235 target, where the simulation shows that the overall behavior of the system is well understood.

Track total ADC - Run1 : U238+U235
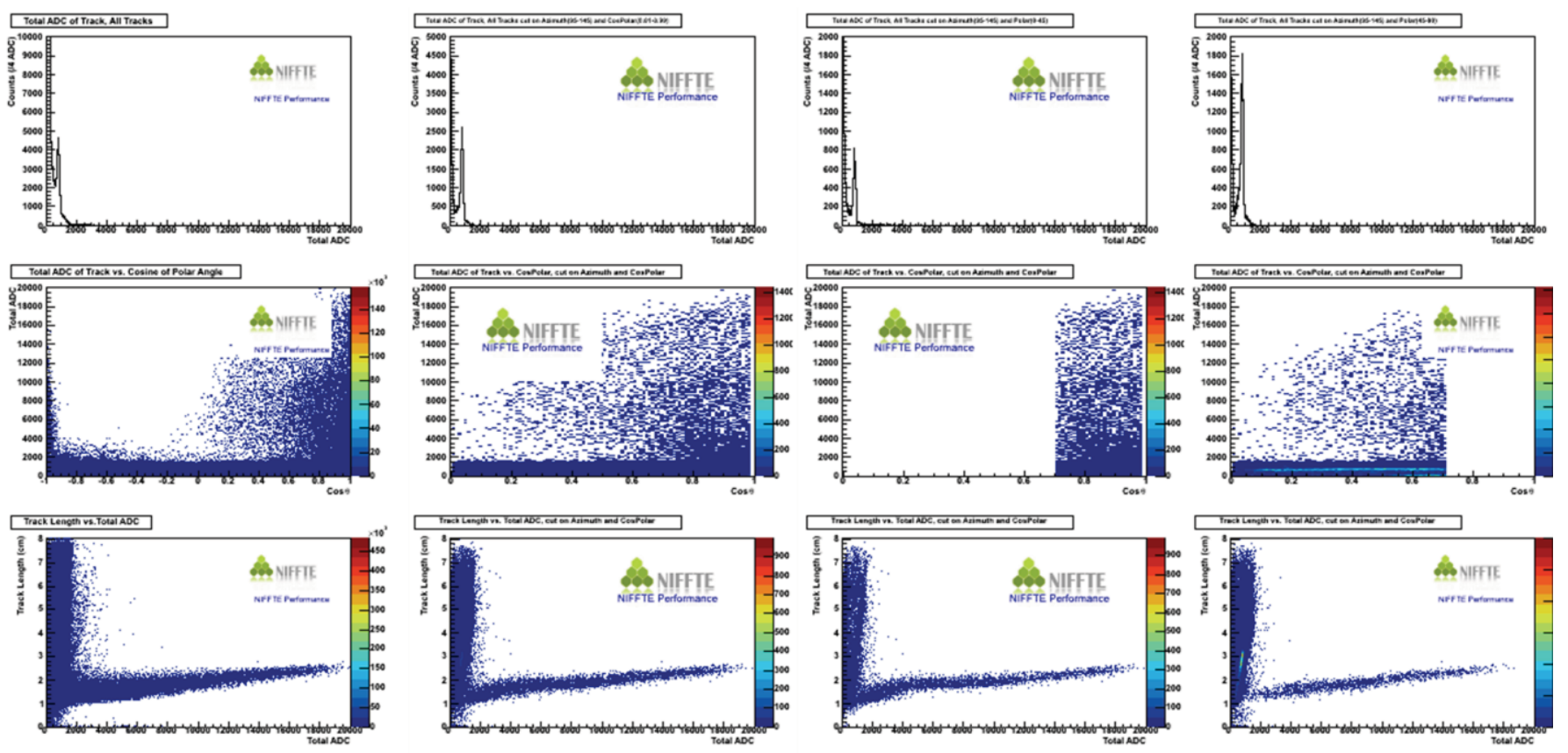

Figure 26: (top to bottom) Total ADC, total ADC vs. polar angle, track length vs. total ADC. (left to right) no angle cuts applied; sextant angle cuts; forward angle cuts; and backward angle cuts (see text). 

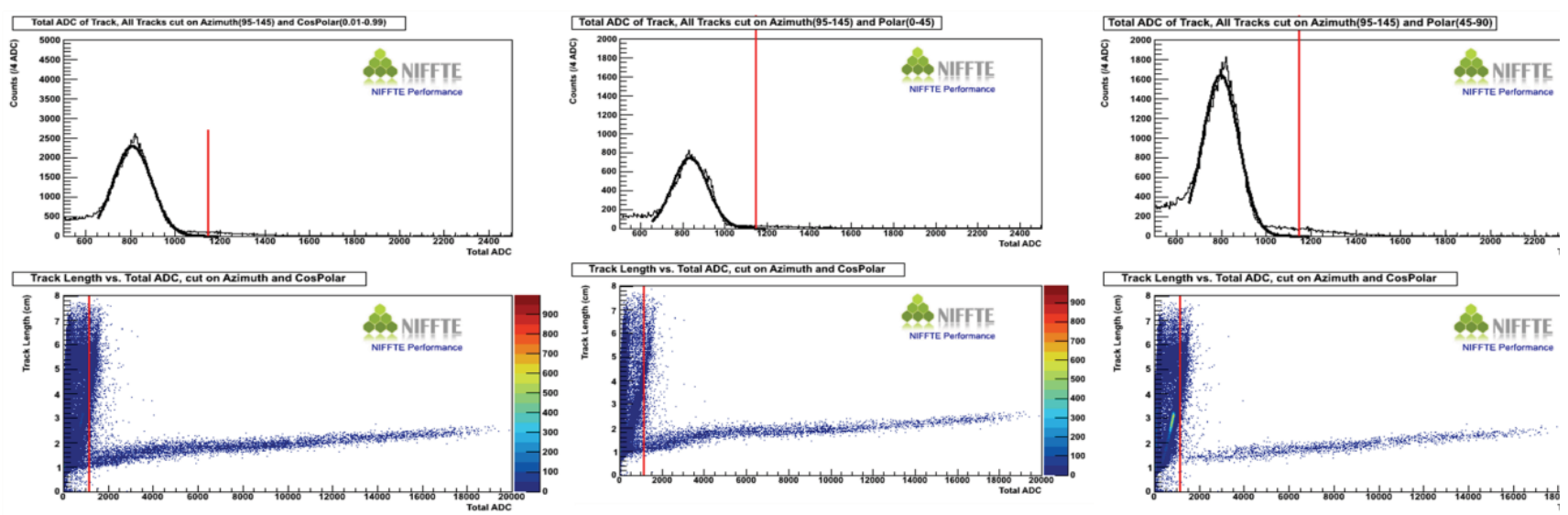

Figure 27: One-dimensional ADC cuts (top) and effect on two-dimensional track length vs. ADC (bottom). (left to right) sextant, forward, and backward angle cuts (see text). These plots demonstrate the source of counting systematics in previous experiments as extrapolations of fission fragments counting in the lower energy region is unreliable.
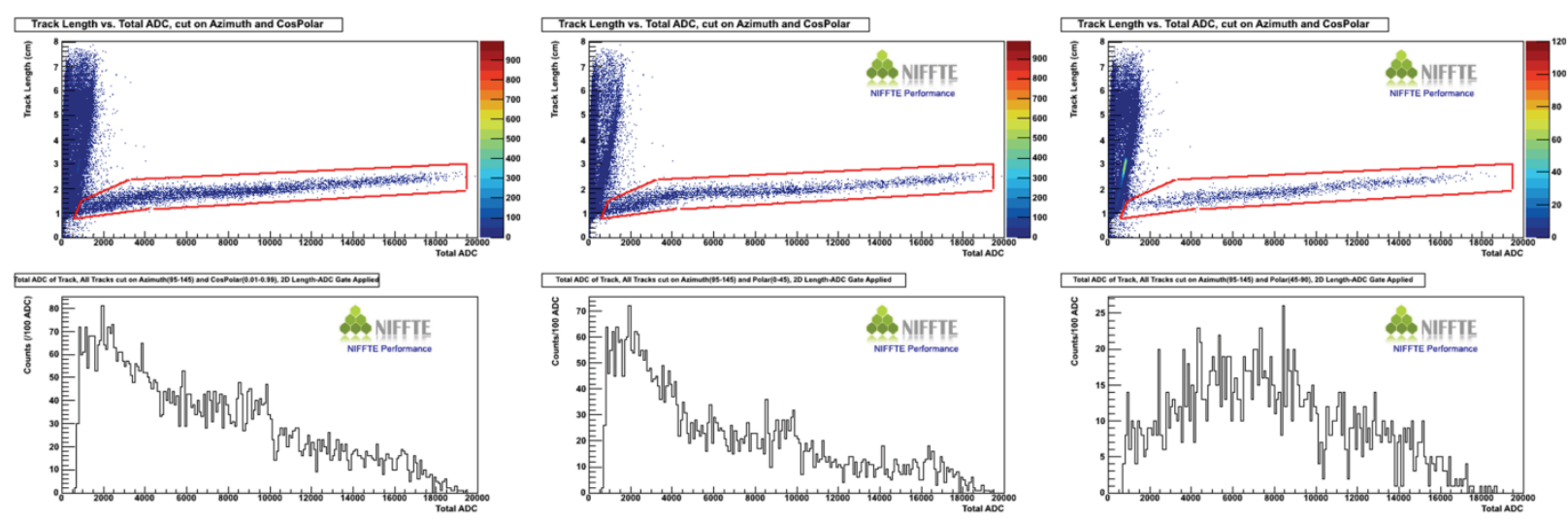

Figure 28: Two-dimensional ADC cuts (top) and effect on one-dimensional total ADC (bottom). (left to right) sextant, forward, and backward angle cuts (see text).
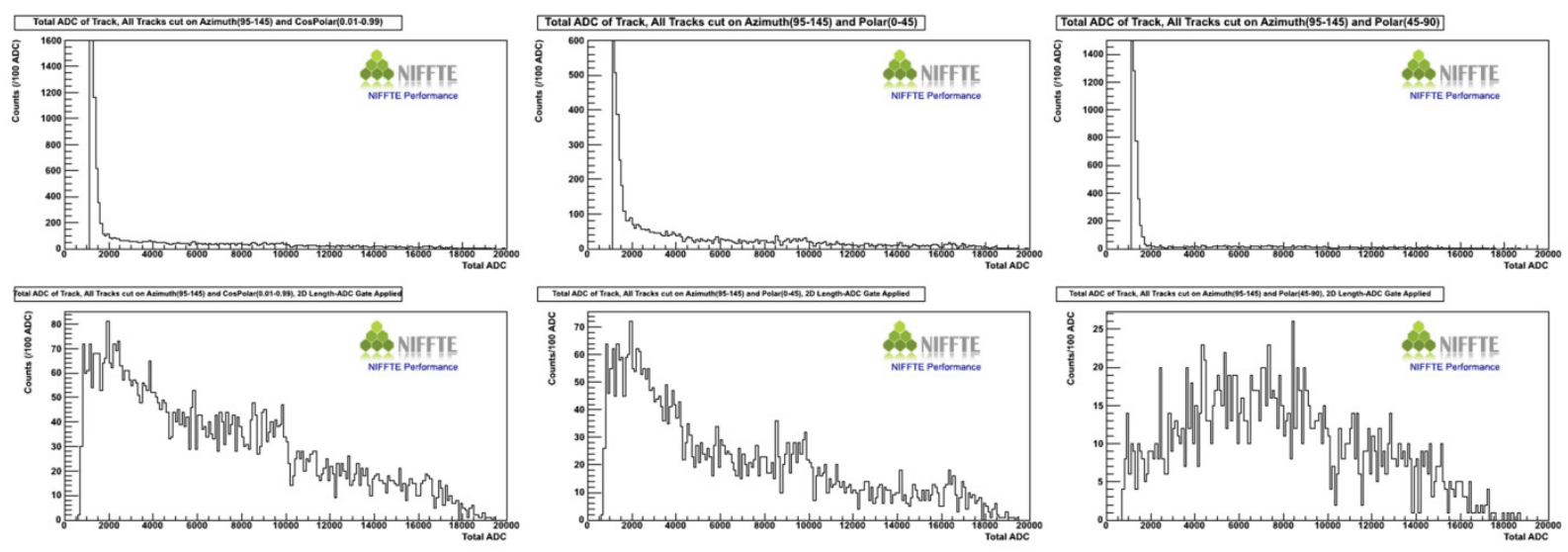

Figure 29: Comparison of one (top) and two (bottom) dimensional cuts on total ADC. (left to right) sextant, forward, and backward angle cuts (see text). 


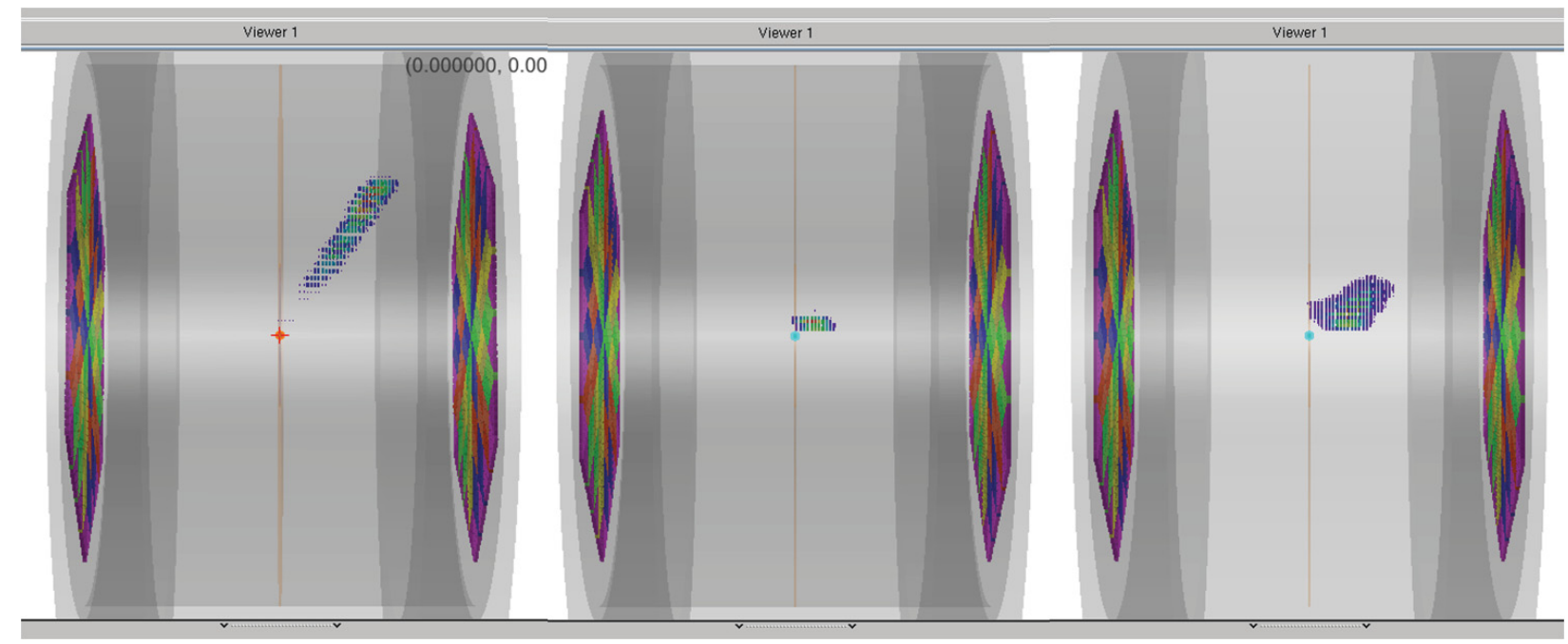

Figure 30: Representative (left) alpha, (center) unidentified, and (right) fission fragment tracks (see text).

\section{Track total ADC - Run2 : U238+U235}
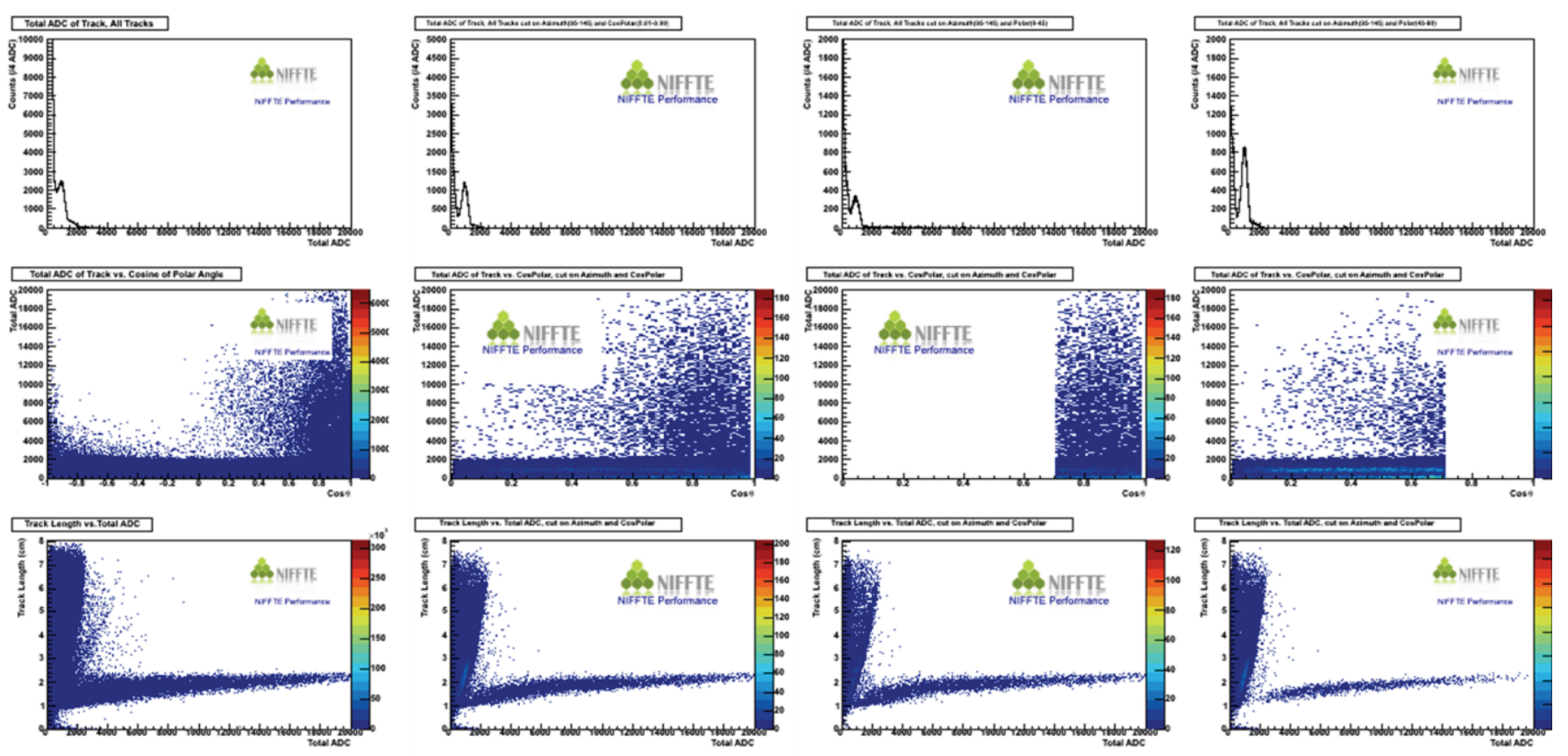

Figure 31: (top to bottom) Total ADC, total ADC vs. polar angle, track length vs. total ADC. (left to right) no angle cuts applied; sextant angle cuts; forward angle cuts; and backward angle cuts (see text). 

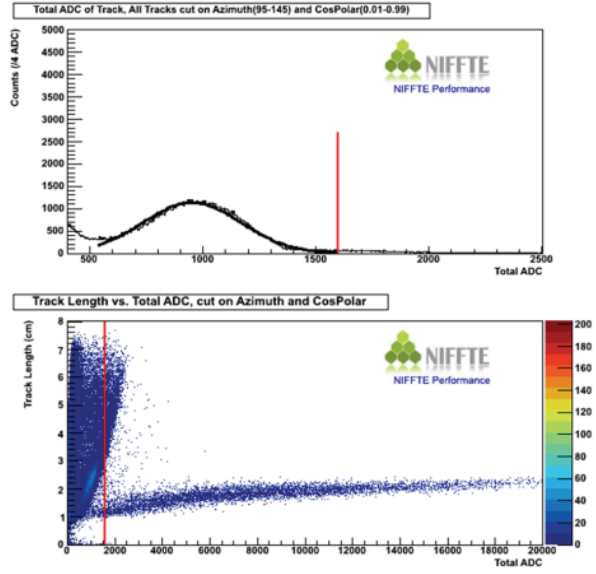
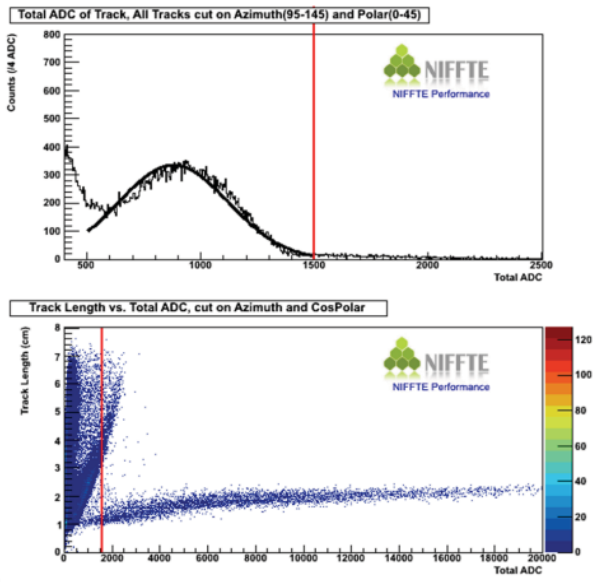
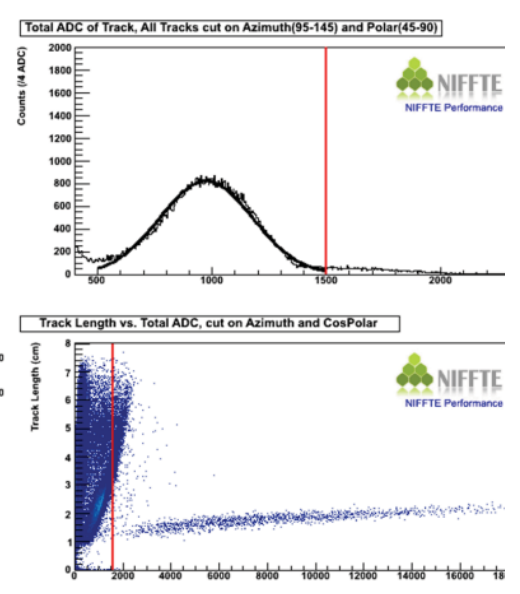

Figure 32: One-dimensional ADC cuts (top) and effect on two-dimensional track length vs. ADC (bottom). (left to right) sextant, forward, and backward angle cuts (see text).
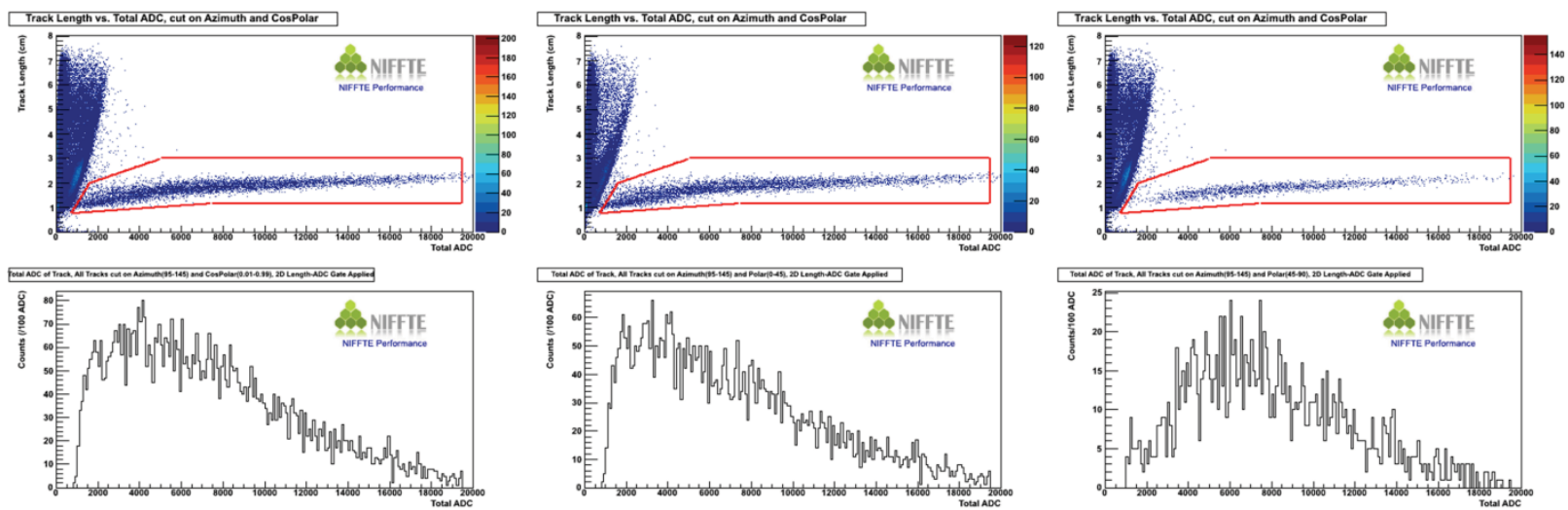

Figure 33: Two-dimensional ADC cuts (top) and effect on one-dimensional total ADC (bottom). (left to right) sextant, forward, and backward angle cuts (see text).
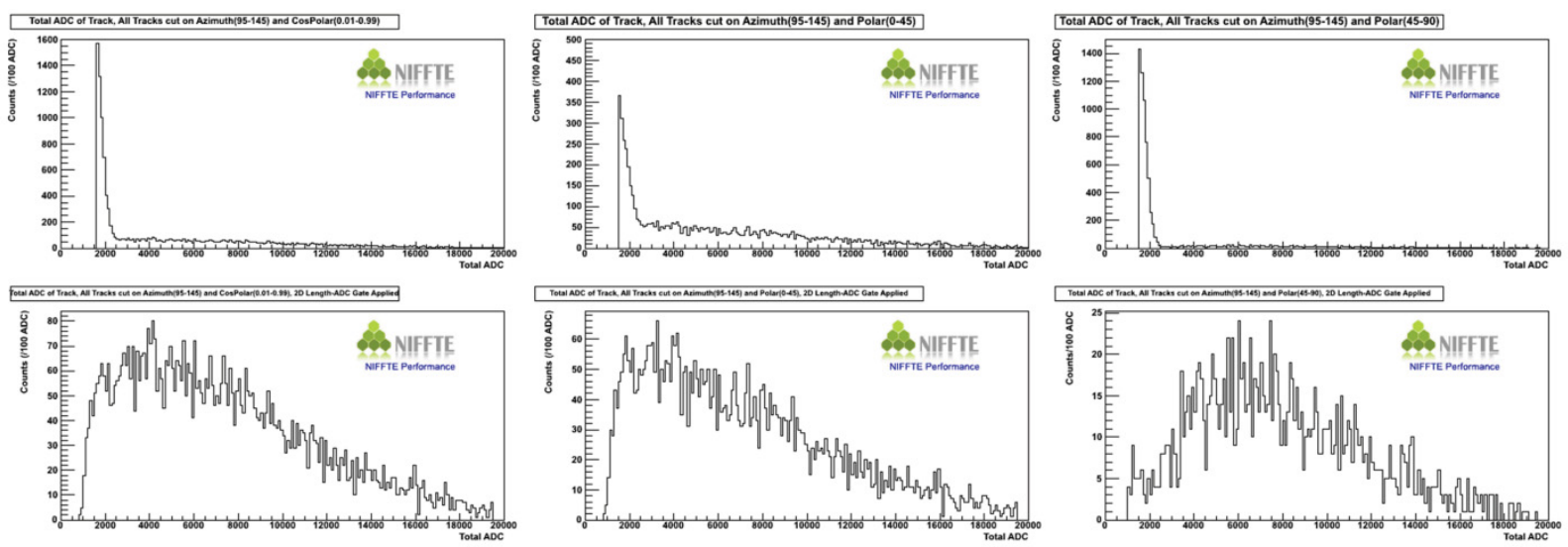

Figure 34: Comparison of one (top) and two (bottom) dimensional cuts on total ADC. (left to right) sextant, forward, and backward angle cuts (see text). 


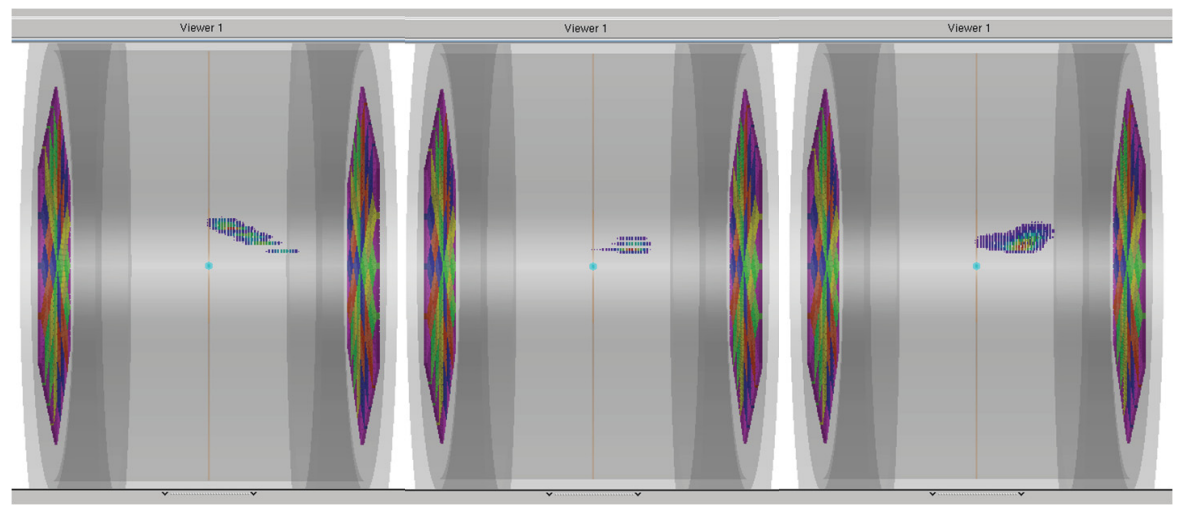

Figure 35: Representative (left) alpha, (center) unidentified, and (right) fission fragment tracks (see text).

The TPC experiment is simulated in a detailed GEANT4 package. The simulations are necessary to insure we understand the response of the system as it is being developed. These detailed simulations provide data nearly identical to that of the actual TPC data stream and are processed through the exact same analysis software for comparisons. Below are plots of the track length versus collected charge for both real data (run 400000487 was a U235 target) and from TPC simulations. The inclusion of the TPC dead channels in the simulation demonstrates that the simulation is performing admirably and also shows that although the particle ID is impacted by dead channels, it is certainly well understood. Of course, the goal is to operate the production TPC with as few dead channels as possible and work continues along that development front. However, the simulations assure us the liabilities will be minimized in any case.

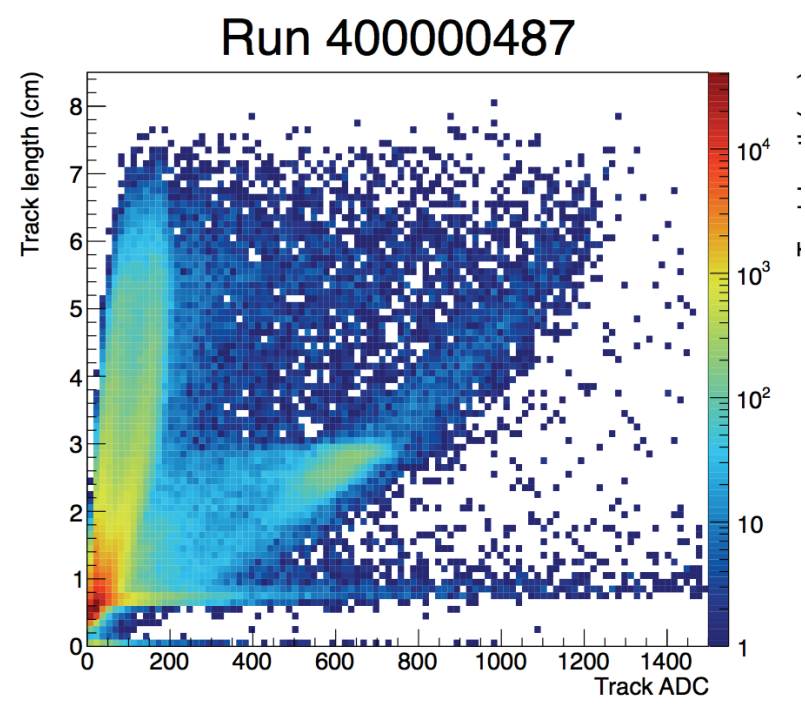

Figure 36: Shown here is reconstructed track length versus uncalibrated charge collection for a single sextant run using $\mathbf{U 2 3 5}$. The protons are the left band, the alphas form the center bands and on this scale, only the tail of the fission fragments is seen as the horizontal band that continues out of view to the right. 


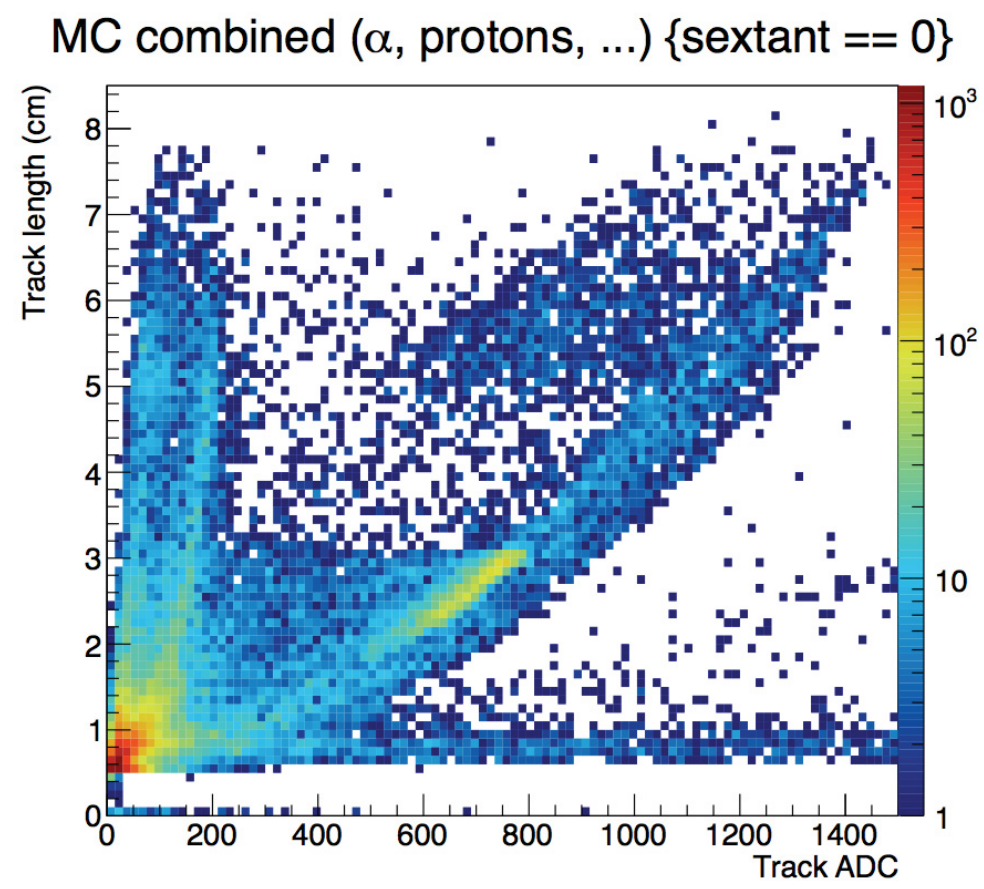

Figure 37:Shown here is the reconstructed track length versus the collected charge in the GEANT4 simulation of the TPC before including the impact of dead channels.

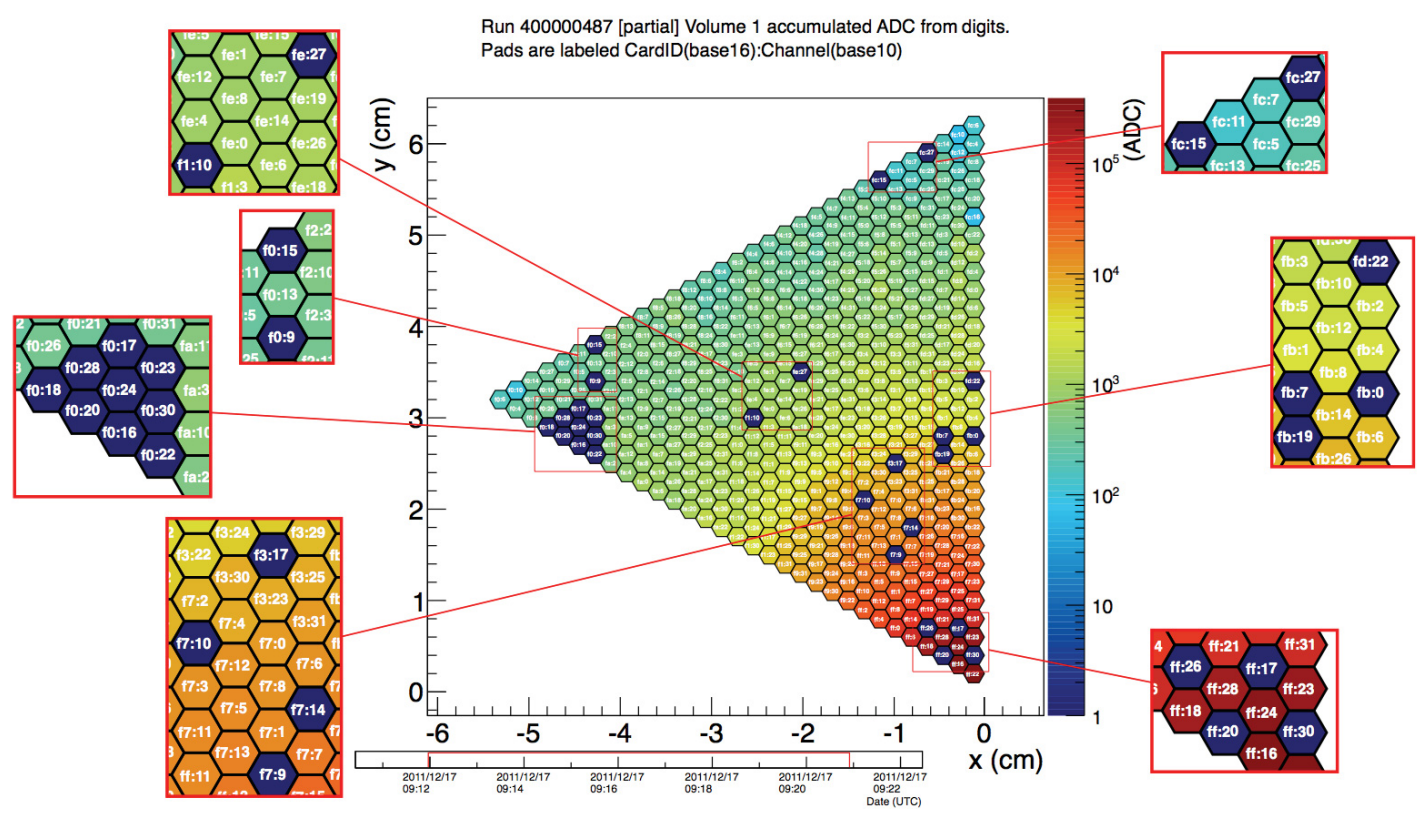

Figure 38: Shown here is the pixel map for the U235 run where the colors represent the total charge collected and the purple pads are those that did not function for this run. 


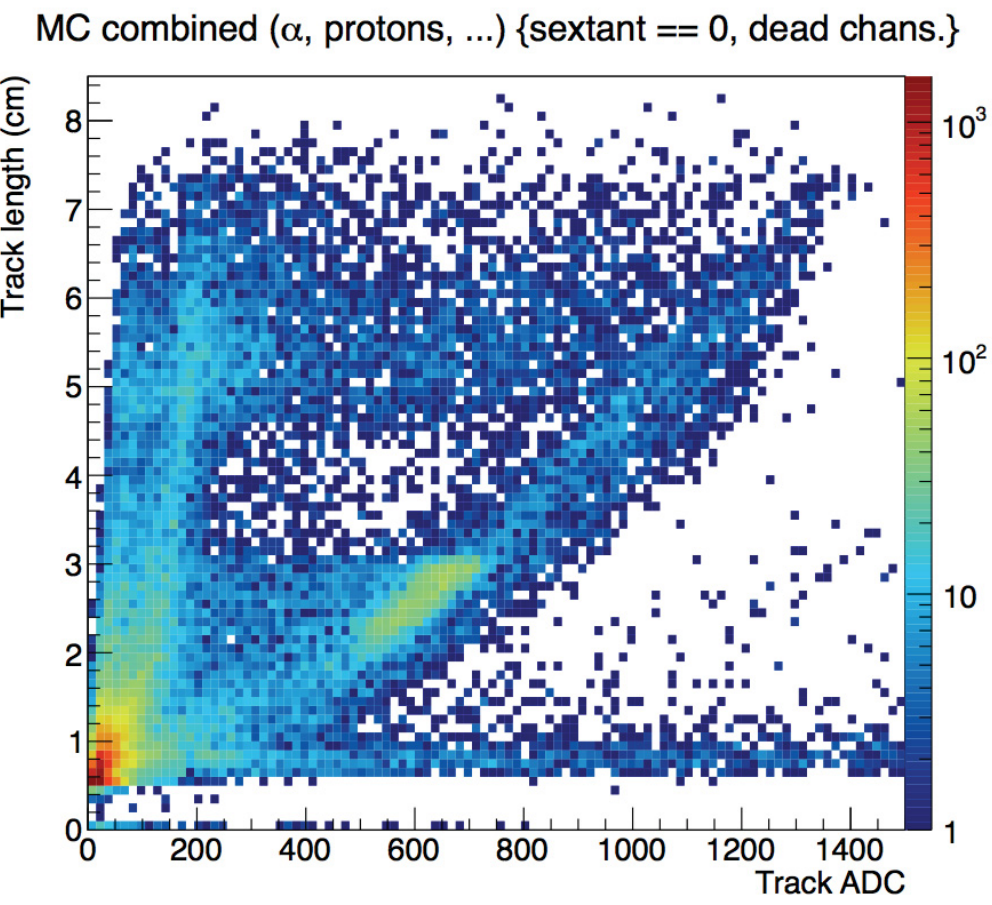

Figure 39: GEANT4 simulations of the TPC run where the impact of the dead channels are included. All pertinent structures in the data are completely recreated, assuring minimal impact on active channel count.

\section{Track total ADC - Full Side TPC U238+U235}

The following data were collected using a fully instrumented side of the TPC at the LANSCE facility. The caveats of the limited viewing restriction of the single sextant data are removed. The power of the TPC particle identification is evident in the simple metric of track length versus uncalibrated charge collected. Several species, along with fission products and alphas can be seen, as they are the result of secondary interactions with the gas (protons) and the other materials in the TPC support structure. As expected, the azimuth angle is flat. The polar angle distribution is shaped due to exclusion of proper calibration constants for the drift speed of the TPC gas. It is clear in both plots that there is very good separation between fission products and alpha particles in the simplest metrics. The overlap in the energy distributions for these particles occurs when fission fragments lose a sizeable fraction of their energy in the target, specifically at grazing angles to the target plane. In the $A D C$ versus polar angle, on can clearly see the fission double hump structure in the uncalibrated drift gas and that the overlap between these and the alpha particles occuring at angles that are nearly coplanar with the target. A simple cut on the polar angle itself will remove all fission product/alpha particle misidentification. 


\section{Track Length vs. ADC}

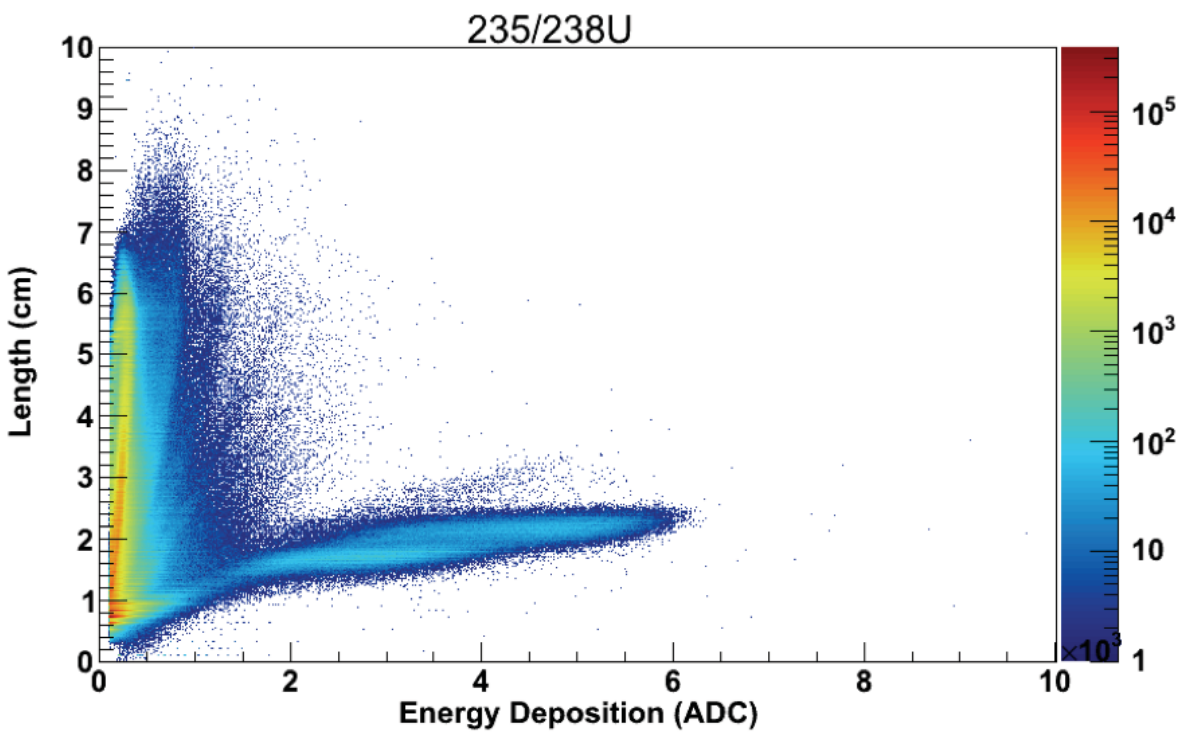

Figure 40: Shown here is the reconstructed track length versus the collected uncalibrated charge. The largest band (upward and on left) is due to alpha particles and protons. The bifurcated distribution to the right is the double humped fission product distribution. The structure between the two are secondary particles from neutrons interacting with other materials in the experiment as well as smearing due to dead channels.

\section{ADC vs. Azimuthal Angle}

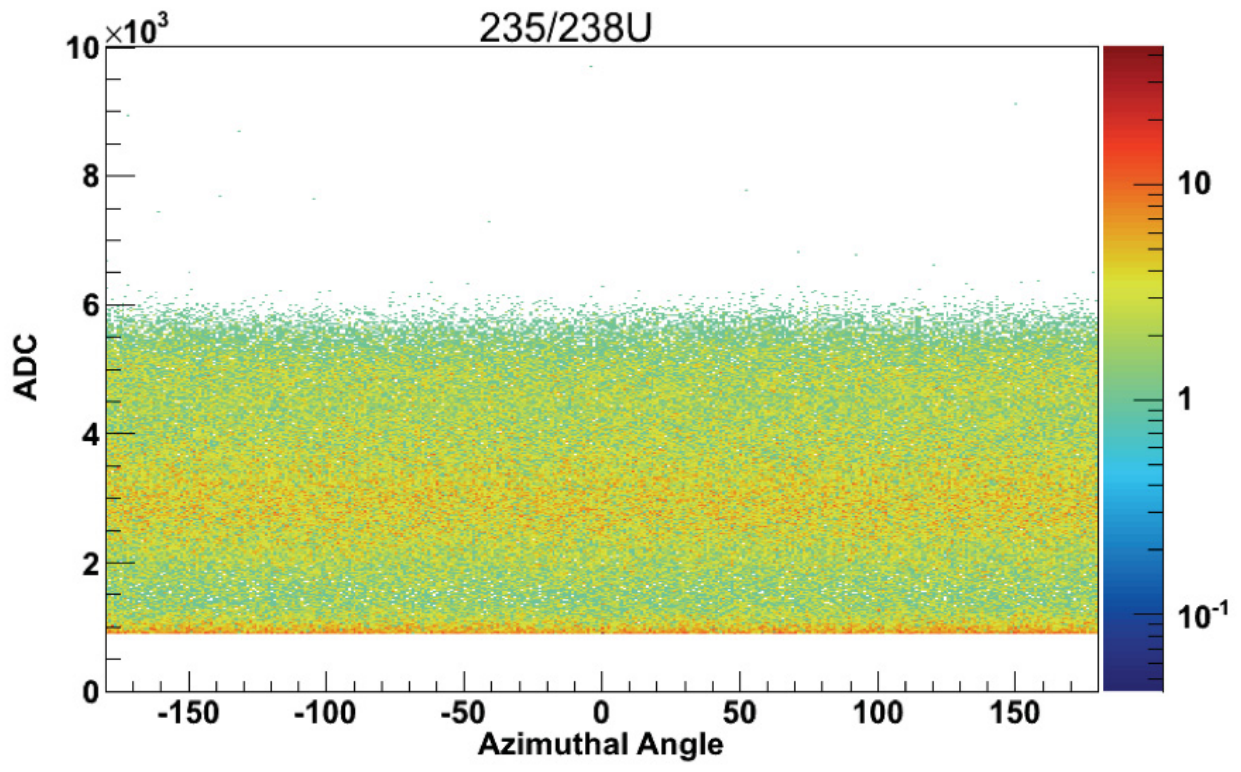

Figure 41: Shown here is the uncalibrated charge collection as a function of azimuthal angle in a fully instrumented side of the TPC. The lower band in ADC is alpha particles and the upper band is fission products. 


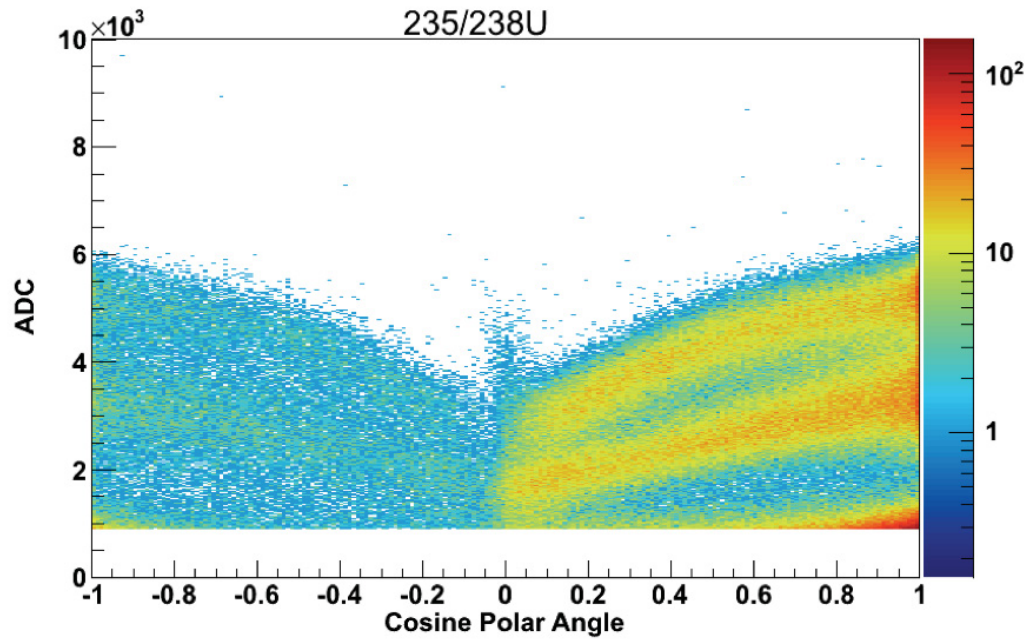

Figure 42: Shown here is the uncalibrated charge collected as a function of the cosine of the track polar angle. In a properly calibrated system, all bands will be flat. The left side of the plot is particles moving in the direction of the beam (secondary interactions and improperly tracked particles from the target). The right side are tracks that move opposite the beam as emitted from the target. The fission double hump is clearly visible and distinct (upper bands) and the only region of overlap between the lower mass fission products and the alphas is near grazing angles to the target, where particles lose increasingly more energy in the target and backing.

\section{Track Length vs. Polar Angle}

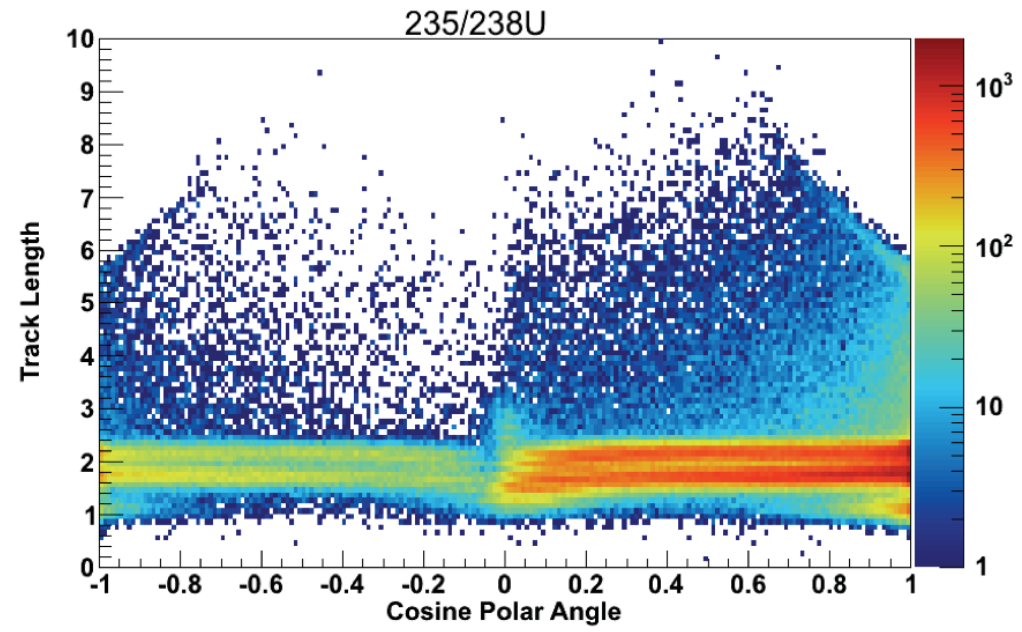

Figure 43: Shown here is the reconstructed track length as a function of the cosine of the polar angle. The left side of the plot is particles moving in either the direction of the beam (secondary interactions or improperly tracked particles from the target). The right side contains tracks that move opposite the beam as emitted from the target. The fission double hump is clearly visible and distinct (lower bands) and the alphas (band near the top) are smeared over a much larger part of this phase space. 


\section{Conclusions}

The current TPC is collecting neutron beam data at the LANSCE WNR facility in Los Alamos with half of the detector instrumented. The system is operating at expected performance levels based on the development approach adopted for this effort that relies heavily on analysis feedback, some of which is presented in this report. The TPC is being tested during the development phase to insure that the required performance goals can be met in the LANSCE beam environment, something that is impossible to simulate in a test bench. This approach has been very fruitful in providing timely and important performance feedback for hardware and software development. The TPC configuration was limited in instrumentation to onesixth of the collection volume in most tests prior to scaling up to half a system, whose benefit can be seen in those data. The capabilities of the partially instrumented TPC is limited and complicated, as was demonstrated in the comparisons. The overall TPC performance is further hampered in analysis by a lack of any, let alone precise, channel by channel calibrations data or the deconvolution of detector behaviors, such as cross talk and charge diffusion. Efforts are underway on all these fronts.

However, it is clear from the initial detailed analyses and simulations, that the particle identification information and vertexing capabilities will meet the needs for reducing or removing these particular systematic uncertainties in the actual production measurements to be carried out in future years, even before the system is completely optimized. 